\title{
GLIVENKO THEOREMS FOR SUBSTRUCTURAL LOGICS OVER FL
}

\author{
NIKOLAOS GALATOS AND HIROAKIRA ONO
}

\begin{abstract}
It is well known that classical propositional logic can be interpreted in intuitionistic propositional logic. In particular Glivenko's theorem states that a formula is provable in the former iff its double negation is provable in the latter. We extend Glivenko's theorem and show that for every involutive substructural logic there exists a minimum substructural logic that contains the first via a double negation interpretation. Our presentation is algebraic and is formulated in the context of residuated lattices. In the last part of the paper, we also discuss some extended forms of the Kolmogorov translation and we compare it to the Glivenko translation.
\end{abstract}

\section{INTRODUCTION}

The following theorem, due to Glivenko [13], shows that classical propositional logic can be interpreted in intuitionistic propositional logic.

Theorem 1.1 (Glivenko). A formula $\phi$ is provable in classical propositional logic iff the formula $\neg \neg \phi$ is provable in intuitionistic propositional logic.

Extensions of this theorem can be found in [5] and [6].

\section{Theorem 1.2.}

(1) A formula $\phi$ is provable in classical propositional logic iff the formula $\neg \neg \phi$ is provable in the extension SBL of Hájek basic logic by the axiom $(\chi \circ(\chi \rightarrow \neg \chi)) \rightarrow \psi$.

(2) A formula $\phi$ is provable in Eukasiewicz infinite-valued logic iff the formula $\neg \neg \phi$ is provable in Hájek basic logic.

(3) Let $\mathbf{L}$ be an extension of $\mathbf{F L}_{\mathbf{e w}}$ - the full Lambek calculus with exchange and weakening, and $0 \rightarrow \psi$ among its axioms - that contains the axiom $\neg \neg(\neg \neg \psi \rightarrow \psi)$ and let $\mathbf{I n}(\mathbf{L})$ be the extension of $\mathbf{L}$ by the axiom $\neg \neg \psi \rightarrow \psi$. Then a formula $\phi$ is provable in $\mathbf{I n}(\mathbf{L})$ iff the formula $\neg \neg \phi$ is provable in $\mathbf{L}$.

Date: October 31, 2005.

1991 Mathematics Subject Classification. Primary: 06F05, Secondary: 08B15, 03B47, 03G10, 03B05, 03B20.

Key words and phrases. Glivenko's theorem, substructural logic, involutive, pointed residuated lattice. 
All the logics mentioned above are special cases of substructural logics over FL; see [11] for a study of these logics. Moreover, the above theorems establish the interpretability of an involutive logic in another logic. A (commutative) logic with negation is called involutive, if the double negation formula $\sim \sim \phi \leftrightarrow \phi$ is provable in it; we use the notation $\sim \phi$ for $\phi \rightarrow 0$, rather than $\neg \phi$, if $0 \rightarrow \psi$ is not assumed to be an axiom. Observe that the previous results are limited to the case, where the rules of exchange and weakening are present. In FL neither of these rules is assumed and two negations $\sim \phi$ and $-\phi$ are considered. In general, a substructural logic is called involutive, if the formulas $-\sim \phi \leftrightarrow \phi$ and $\sim-\phi \leftrightarrow \phi$ are provable.

If $\mathbf{K}$ and $\mathbf{L}$ are substructural logics, we say that the Glivenko property holds for $\mathbf{K}$ relative to $\mathbf{L}$ iff, for all formulas $\phi$ over the language of $\mathbf{F L}$,

$$
\vdash_{\mathbf{L}} \phi \text { iff } \vdash_{\mathbf{K}}-\sim \phi \text { iff } \vdash_{\mathbf{K}} \sim-\phi,
$$

where $\vdash_{\mathbf{M}}$ denotes the consequence relation associated with the logic $\mathbf{M}$.

We will base our study on results of [11] that are reviewed in the next section. In view of the fact that the subvarieties of the variety $\mathcal{F} \mathcal{L}$ of pointed residuated lattices serve as equivalent algebraic semantics for substructural logics over FL - see Theorem 2.5 - the Glivenko property can also be reformulated in algebraic terms. If $\mathcal{W}$ and $\mathcal{V}$ are subvarieties of $\mathcal{F} \mathcal{L}$, we say that the Glivenko property holds for $\mathcal{W}$ relative to $\mathcal{V}$ iff, for every term $t$ over the language of $\mathcal{F} \mathcal{L}$,

$$
\models_{\mathcal{V}} 1 \leq t \text { iff } \mid \models_{\mathcal{W}} 1 \leq-\sim t \text { iff } \models_{\mathcal{W}} 1 \leq \sim-t,
$$

where $\models_{\mathcal{K}}$ denotes the semantical consequence relation relative to the class of algebras $\mathcal{K}$; e.g., see section 3.2 of [11]. It follows from the algebraization of $\mathbf{F L}$ that the Glivenko property holds for $\mathbf{K}$ relative to $\mathbf{L}$ iff it holds for $\mathcal{V}(\mathbf{K})$ relative to $\mathcal{V}(\mathbf{L})$.

It is natural to consider the following strengthening of the Glivenko property. If $\mathcal{W}$ and $\mathcal{V}$ are subvarieties of $\mathcal{F} \mathcal{L}$, we say that the equational Glivenko property holds for $\mathcal{W}$ relative to $\mathcal{V}$ iff, for all terms $s, t$ over the language of $\mathcal{F} \mathcal{L}$

$$
\models \mathcal{V} s \leq t \text { iff } \mid=\mathcal{W}-\sim s \leq-\sim t \text { iff } \models \mathcal{W} \sim-s \leq \sim-t .
$$

On the other hand, staying within the setting of logic, we can strengthen the Glivenko property in a different direction. We say that the deductive Glivenko property holds for $\mathbf{K}$ relative to $\mathbf{L}$ iff, for every set of formulas $\Sigma \cup\{\phi\}$ over the language of $\mathbf{F L}$,

$$
\Sigma \vdash_{\mathbf{L}} \phi \text { iff }-\sim \Sigma \vdash_{\mathbf{K}}-\sim \phi \text { iff } \sim-\Sigma \vdash_{\mathbf{K}} \sim-\phi,
$$

where $-\sim \Sigma=\{-\sim \sigma \mid \sigma \in \Sigma\}$. In algebraic terms, the deductive Glivenko property holds for $\mathcal{W}$ relative to $\mathcal{V}$ iff, for all sets of terms $\left\{t_{i} \mid \in I\right\} \cup\{t\}$ over the language of $\mathcal{F} \mathcal{L}$,

$$
D \models_{\mathcal{V}} 1 \leq t \text { iff }-\sim D \models_{\mathcal{W}} 1 \leq-\sim t \text { iff } \sim-D \models_{\mathcal{W}} 1 \leq \sim-t,
$$

where $D=\left\{1 \leq t_{i} \mid \in I\right\}$ and $-\sim D=\left\{1 \leq-\sim t_{i} \mid i \in I\right\}$.

A common strengthening of all these three types of Glivenko property is the following. If $\mathcal{W}, \mathcal{V}$ are subvarieties of $\mathcal{F} \mathcal{L}$, we say that the deductive 
equational Glivenko property holds for $\mathcal{W}$ relative to $\mathcal{V}$ iff, for all sets of equations $E \cup\{s \approx t\}$ over the language of $\mathcal{F} \mathcal{L}$,

$$
E \mid \mathcal{V} s \leq t \text { iff }-\sim E \models \mathcal{W}-\sim s \leq-\sim t \text { iff } \sim-E \models \mathcal{W} \sim-s \leq \sim-t,
$$

where $-\sim E=\{-\sim u \approx-\sim v \mid(u \approx v) \in E\}$.

We will prove that the equational Glivenko property and the deductive equational Glivenko property are equivalent and they imply that $\mathbf{L}$ (or $\mathcal{V}$ ) is involutive; a variety $\mathcal{V}$ is called involutive, if it satisfies $\sim-x \approx x \approx-\sim x$. The other properties are not equivalent in general - see Proposition 4.9 and Proposition 4.10 - but under the assumption that $\mathbf{L}$ ( or $\mathcal{V}$ ) is involutive all of the properties mentioned above are equivalent.

We show that for every involutive substructural logic $\mathbf{L}$, there exists a substructural logic $\mathbf{G}(\mathbf{L})$ such that some/any Glivenko property holds for a substructural logic $\mathbf{K}$ relative to $\mathbf{L}$ iff $\mathbf{G}(\mathbf{L}) \subseteq \mathbf{K} \subseteq \mathbf{L}$, see Corollary 4.7. Given an axiomatization of a logic $\mathbf{L}$, we provide an axiomatization for the logic $\mathbf{G}(\mathbf{L})$, see Corollary 4.12 , which is finite if $\mathbf{L}$ is finitely axiomatized, see Corollary 4.13, and show that $\mathbf{G}$ is an interior operator on the lattice of substructural logics, see Lemma 3.3(1). This answers the question: Given an involutive substructural logic $\mathbf{L}$, for which logics does some Glivenko property hold relative to $\mathbf{L}$ ?

We continue by addressing a question in the other direction: Given a substructural logic $\mathbf{K}$, when and relative to which logics does a Glivenko property hold for $\mathbf{K}$ ? We call two substructural logics (or subvarieties of $\mathcal{F} \mathcal{L}$ ) Glivenko equivalent if they contain the same negated formulas (equations, respectively), see Lemma 3.1. The equivalence classes are intervals of the lattice of substructural logics (the lattice of subvarieties of $\mathcal{F} \mathcal{L}$ ) of the form $[\mathbf{G}(\mathbf{K}), \mathbf{M}(\mathbf{K})]$ (or $[\mathbf{M}(\mathcal{W}), \mathbf{G}(\mathcal{W})]$ ). It turns out that two logics $\mathbf{K}_{1}$ and $\mathbf{K}_{2}$ are Glivenko equivalent iff $\mathbf{G}\left(\mathbf{K}_{1}\right)=\mathbf{G}\left(\mathbf{K}_{2}\right)$; see Lemma 3.3(3). In this case, any of the Glivenko properties holds for $\mathbf{K}_{2}$ relative to some logic iff the property holds for $\mathbf{K}_{1}$ relative to the same logic. Additionally, any of the Glivenko properties above holds for $\mathbf{K}$ relative to some substructural logic iff it holds relative to $\mathbf{M}(\mathbf{K})$; see Propositions 4.1, 4.4 and 4.6.

For each of the three Glivenko properties, we describe different degrees of involutiveness that $\mathbf{M}(\mathbf{K})$ has to possess. The existence of an involutive substructural logic (or subvariety of $\mathcal{F} \mathcal{L}$ ) relative to which some/any of the Glivenko properties holds for $\mathbf{K}$ (or $\mathcal{W}$ ) is equivalent to the condition that $\mathbf{K}$ contains the Glivenko logic $\mathbf{G}$ (or $\mathcal{W}$ being contained in the Glivenko variety $\mathcal{G}$ ); see Theorem 5.7 and the comments following it.

In each of the three Glivenko properties discussed above, there are three statements involved, which are stipulated to be equivalent. For example, we have

$$
\models_{\mathcal{V}} s \leq t \text { iff } \mid \models_{\mathcal{W}}-\sim s \leq-\sim t \text { iff } \models_{\mathcal{W}} \sim-s \leq \sim-t
$$


in the equational Glivenko property. One can consider finer versions of the properties by stipulating that the first two statements (left version) or the first and the last statement (right version of the property) are equivalent. So, for example, the left equational Glivenko property holds for $\mathcal{W}$ relative to $\mathcal{V}$ iff, for all terms $s, t$ over the language of $\mathcal{F} \mathcal{L}$,

$$
\models_{\mathcal{V}} s \leq t \text { iff } \models_{\mathcal{W}}-\sim s \leq-\sim t
$$

Our analysis respects these more detailed considerations and provides left and right versions of each of the results that we show. For simplicity we state most of the results in their left versions, but the opposite statements, where "left" is replaced by "right" and the terms and equations in the statement are replaced by their opposite, hold, as well.

In Section 6, we present some special cases. In particular, we show that Theorems 1.1 and 1.2 follow from our analysis. Moreover, we obtain simplified axiomatizations for the largest integral subvariety of $\mathcal{F} \mathcal{L}$ for which Glivenko's theorem holds with respect to a given integral involutive variety. Also, we study the case where a Glivenko property holds relative to the variety of Boolean algebras and the case of the subvarieties of GBL-algebras.

Finally, in Section 7, we discuss briefly a translation that generalizes the Kolmogorov translation to substructural logics and compare it to the Glivenko translation.

\section{Preliminaries}

Our approach, presentation and proofs of most of the results are algebraic. Therefore, we begin by reviewing the definitions and results of [11] needed for this paper, pertaining to the connection of substructural logics to their algebraic counterparts: residuated lattices. The reader is referred to [11] for the proofs and for further results.

A residuated lattice-ordered monoid, or residuated lattice, is an algebra $\mathbf{A}=\langle A, \wedge, \vee, \cdot, \backslash, /, 1\rangle$ such that $\langle A, \wedge, \vee\rangle$ is a lattice, $\langle A, \cdot, 1\rangle$ is a monoid and multiplication is residuated with respect to the order by the division operations $\backslash$, /; i.e., for all $a, b, c \in A$,

$$
a \cdot b \leq c \Leftrightarrow a \leq c / b \Leftrightarrow b \leq a \backslash c .
$$

A pointed residuated lattice, or $F L$-algebra, $\mathbf{A}=\langle A, \wedge, \vee, \cdot, \backslash, /, 1,0\rangle$ is an algebra such that $\langle A, \wedge, \vee, \cdot, \backslash, /, 1\rangle$ is a residuated lattice and 0 is an arbitrary element of $A$.

We assume that, among the operations for residuated lattices, multiplication is performed first followed by the division operations and the lattice operations. So, for example, $x / y z \wedge u \backslash v$ simplifies $[x /(y z)] \wedge(u \backslash v)$. We denote by $F m_{\mathcal{L}}$ the set of terms over the language $\mathcal{L}$ of residuated lattices. By $t \leq s$ we denote both the equality $t=t \wedge s$, if $t, s$ are elements of a (pointed) residuated lattice, and the equation $t \approx t \wedge s$, if $t, s$ are terms. It is easy to see that in a (pointed) residuated lattice the equality 
$s=t$ is equivalent to the inequality $1 \leq s \backslash t \wedge t \backslash s$. The opposite $t^{\text {op }}$ of a (pointed) residuated lattice term $t$ is defined inductively on the complexity of $t$. For all terms $s, t$, we define $1^{\mathrm{op}}=1,0^{\mathrm{op}}=0,(s \cdot t)^{\mathrm{op}}=t \cdot s$, $(s \backslash t)^{\mathrm{op}}=t / s,(t / s)^{\mathrm{op}}=s \backslash t,(s \wedge t)^{\mathrm{op}}=t \wedge s$, and $(s \vee t)^{\mathrm{op}}=t \vee s$. Essentially, the opposite of a term is its "mirror image". We extend the definition to equations, by $(s \approx t)^{\mathrm{op}}=\left(t^{\mathrm{op}} \approx s^{\mathrm{op}}\right)$, and to metalogical statements in the obvious way. Note that $(s \leq t)^{\mathrm{op}}=\left(t^{\mathrm{op}} \geq s^{\mathrm{op}}\right)$. Examples of mutually opposite equations can be seen in each statement of the following lemma. If $\mathbf{A}=\langle\wedge, \vee, \cdot, \backslash, /, 1,0\rangle$ is a pointed residuated lattice, the algebra $\mathbf{A}^{\mathrm{op}}=\left\langle\wedge, \vee,{ }^{\mathrm{op}}, /, \backslash, 1,0\right\rangle$, where $x \cdot{ }^{\mathrm{op}} y=y \cdot x$, is also a pointed residuated lattice that is called the opposite of $\mathbf{A}$.

Lemma 2.1. [3], [11] The following identities hold in all (pointed) residuated lattices.

(1) $x(y \vee z) \approx x y \vee x z$ and $(y \vee z) x \approx y x \vee z x$

(2) $x \backslash(y \wedge z) \approx(x \backslash y) \wedge(x \backslash z)$ and $(y \wedge z) / x \approx(y / x) \wedge(z / x)$;

(3) $x /(y \vee z) \approx(x / y) \wedge(x / z)$ and $(y \vee z) \backslash x \approx(y \backslash x) \wedge(z \backslash x)$;

(4) $(x / y) y \leq x$ and $y(y \backslash x) \leq x$;

(5) $x(y / z) \leq(x y) / z$ and $(z \backslash y) x \leq z \backslash(y x)$;

(6) $(x / y) / z \approx x /(z y)$ and $z \backslash(y \backslash x) \approx(y z) \backslash x$;

(7) $x \backslash(y / z) \approx(x \backslash y) / z$;

(8) $x / 1 \approx x \approx 1 \backslash x$;

(9) $1 \leq x / x$ and $1 \leq x \backslash x$;

(10) $x \leq y /(x \backslash y)$ and $x \leq(y / x) \backslash y$;

(11) $y /((y / x) \backslash y)=y / x$ and $(y /(x \backslash y)) \backslash y=x \backslash y$;

(12) $x /(x \backslash x)=x$ and $(x / x) \backslash x=x$;

(13) $(z / y)(y / x) \leq z / x$ and $(x \backslash y)(y \backslash z) \leq x \backslash z$.

Multiplication is order preserving in both coordinates; each division operation is order preserving in the numerator and order reversing in the denominator. Moreover, if a residuated lattice has a least element $\perp$, then it has a greatest element $\top$, as well, and $\top=\perp / \perp=\perp \backslash \perp$.

The class $\mathcal{R} \mathcal{L}$ of residuated lattices and the class $\mathcal{F} \mathcal{L}$ of pointed residuated lattices are both varieties. We denote their subvariety lattices by $\mathbf{S}(\mathcal{R} \mathcal{L})$ and $\mathbf{S}(\mathcal{F} \mathcal{L})$, respectively.

A (pointed) residuated lattice is called commutative, if its monoid reduct is commutative; i.e., if it satisfies the identity $x y \approx y x$. It is called integral, if its lattice reduct has a top element and the latter coincides with the multiplicative identity 1 ; i.e., if it satisfies $x \leq 1$. Finally, it is called contractive, if it satisfies the identity $x \leq x^{2}$. It is easy to see that in a residuated lattice commutativity is equivalent to $x / y \approx y \backslash x$; in this context we write $x \rightarrow y$ for $x \backslash y$. We denote by $\mathcal{C} \mathcal{R} \mathcal{L}$ and $\mathcal{C} \mathcal{F} \mathcal{L}$ the varieties of commutative residuated lattices and pointed commutative residuated lattices, respectively.

In a pointed residuated lattice we define two negation operations $\sim x=$ $x \backslash 0$ and $-x=0 / x$. A pointed residuated lattice is called left involutive 
(right involutive), if it satisfies the identity $-\sim x \approx x(\sim-x \approx x$, respectively). It is called involutive, if it is both left and right involutive; it is called cyclic, if it satisfies $\sim x \approx-x$. Note that for every pointed residuated lattice term $t,(\sim t)^{\mathrm{op}}=-t^{\mathrm{op}}$ and $(-t)^{\mathrm{op}}=\sim t^{\mathrm{op}}$ and that every commutative pointed residuated lattice is cyclic. We assume that the negation operations have priority over all other operations; for example, $-y / x$ means $(-y) / x$.

Lemma 2.2. If $\mathbf{A}$ is a pointed residuated lattice and $x, y$ in $A$, then

(1) $\sim(x \vee y)=\sim x \wedge \sim y$ and $-(x \vee y)=-x \wedge-y$;

(2) if $x \leq y$, then $\sim y \leq \sim x$ and $-y \leq-x$;

(3) $x \leq-\sim x$ and $x \leq \sim-x$;

(4) $\sim-\sim x=\sim x$ and $-\sim-x=-x$;

(5) $-\sim x=-\sim y$ iff $\sim x=\sim y$.

(6) $\sim-x=\sim-y$ iff $-x=-y$.

(7) $-\sim(x / y) \cdot y \leq-\sim x$ and $y \cdot \sim-(y \backslash x) \leq \sim-x$.

(8) $-y / x=-(x y)$ and $x \backslash \sim y=\sim(y x)$.

(9) $x \backslash-y=\sim x / y$.

(10) $x \backslash-y=-\sim x \backslash-y$ and $\sim y / x=\sim y / \sim-x$.

(11) $-\sim(x \backslash y) \leq-\sim(-\sim x \backslash-\sim y)$ and $\sim-(y / x) \leq \sim-(\sim-y / \sim-x)$.

(12) $-\sim(-\sim y / x)=-\sim y / x$ and $\sim-(x \backslash \sim-y)=x \backslash \sim-y$.

(13) $x y \leq-z$ iff $-\sim x \cdot y \leq-z$. Also, $x y \leq \sim z$ iff $x \cdot \sim-y \leq \sim z$.

Proof. The first statement is a direct consequence of Lemma 2.1(3); the second statement follows from the first one. Statements (3) and (4) are consequences of statements (10) and (11) in Lemma 2.1, for $y=0$. Moreover, (5) and (6) follow from (4). For (7), note that $(x / y) y(x \backslash 0) \leq x(x \backslash 0) \leq 0$, by (4) of Lemma 2.1. We have successively, $y(x \backslash 0) \leq(x / y) \backslash 0=[0 /((x / y) \backslash 0)] \backslash 0$, by $(4),[0 /((x / y) \backslash 0)] y(x \backslash 0) \leq 0,[0 /((x / y) \backslash 0)] y \leq 0 /(x \backslash 0)$, and $-\sim(x / y)$. $y \leq-\sim x$. Likewise, we prove the opposite identity. To obtain (8), note that, by (6) of Lemma 2.1, we have $-y / x=(0 / y) / x=0 / x y=-(x y)$. For (9), we use (7) of Lemma 2.1 to obtain $x \backslash-y=x \backslash(0 / y)=(x \backslash 0) / y=\sim x / y$. Using (9) and (4), we have $x \backslash-y=\sim x / y=\sim-\sim x / y=-\sim x \backslash-y$, so we obtain (10). By (3) and (10), $x \backslash y \leq x \backslash-\sim y=-\sim x \backslash-\sim y$; then, (11) follows by (2). For (12), we use (8) and (4) to obtain $-\sim(-\sim y / x)=-\sim-(x$. $\sim y)=-(x \cdot \sim y)=-\sim y / x$. Finally, for (13) we have $x y \leq-z=0 / z$ iff $x y z \leq 0$ iff $y z \leq x \backslash 0=-\sim x \backslash 0$ iff $-\sim x \cdot y z \leq 0$ iff $-\sim x \cdot y \leq-z$.

Lemma 2.3. If $\mathbf{A}$ is a cyclic pointed residuated lattice, then for all $x, y, z \in$ $A$, we have

(1) $x y \leq 0$ iff $y x \leq 0$,

(2) $x y z \leq 0$ iff $y z x \leq 0$ iff $z x y \leq 0$,

(3) $x y z \leq 0$ iff $\sim \sim x \cdot \sim \sim y \cdot \sim \sim z \leq 0$,

(4) $x y \leq \sim z$ iff $\sim \sim x \cdot \sim \sim y \leq \sim z$.

Proof. For (1), if $x y \leq 0$, then $x \leq 0 / y=y \backslash 0$, so $y x \leq 0$. Condition (2) is a direct consequence of (1). 
For (3), note that $\sim \sim x \cdot \sim \sim y \cdot \sim \sim z \leq 0$ implies $x y z \leq 0$, by Lemma 2.2(3). Conversely, assume that $x y z \leq 0$. Then, we have $y z \leq x \backslash 0=\sim x=\sim \sim \sim x$, by Lemma 2.2(4). So, we obtain $\sim \sim x \cdot y \cdot z \leq 0$ and $y \cdot z \cdot \sim \sim x \leq 0$, by (2). By repeating the same argument twice, we obtain $\sim \sim x \cdot \sim \sim y \cdot \sim \sim c \leq 0$.

Finally, (4) follows easily from (3).

Let $\mathcal{L}=\{\wedge, \vee, \cdot, \backslash, /, 1,0\}$ be the language of pointed residuated lattices. By FL we denote the full Lambek sequent calculus over $\mathcal{L}$, as well as the set of formulas provable in it; see [11] for the list of the rules of FL.

If $\Sigma \cup\{\phi\}$ is a set of formulas, we write $\Sigma \vdash_{\mathbf{F L}} \phi$ in case there is a proof of the sequent $\Rightarrow \phi$ in the system obtained from FL by adding as initial sequents the ones of the form $\Rightarrow \sigma$, for $\sigma \in \Sigma$. Clearly, $\vdash_{\mathbf{F L}}$ is a substitution invariant and finitary consequence relation on $\mathbf{F} \mathbf{m}_{\mathcal{L}}$; for the definition of consequence relations and their properties, see e.g. [8].

A substructural logic (over FL) is a set of formulas that is closed under $\vdash_{\mathbf{F L}}$ and substitution. If $\mathbf{L}$ is a substructural logic and $\Sigma \cup\{\phi\}$ a set of formulas, we write $\Sigma \vdash_{\mathbf{L}} \phi$ for $\Sigma \cup \mathbf{L} \vdash_{\mathbf{F L}} \phi$. It is clear that $\vdash_{\mathbf{L}}$ is a substitution invariant and finitary consequence relation. We say that a substructural $\operatorname{logic} \mathbf{L}$ is axiomatized by a set of formulas $\Sigma$, if $\mathbf{L}$ is the smallest substructural logic containing $\Sigma$.

Theorem 2.4. [11] A set of formulas $\mathbf{L}$ is a substructural logic iff it is closed under substitution and under the following rules

(fl) $\quad \mathbf{F L} \subseteq \mathbf{L}$.

$\left(m p_{\ell}\right) \quad$ If $\phi, \phi \backslash \psi \in \mathbf{L}$, then $\psi \in \mathbf{L}$.

(adju) If $\phi \in \mathbf{L}$, then $\phi \wedge 1 \in \mathbf{L}$.

(pn) If $\phi \in \mathbf{L}$, then $\psi \backslash \phi \psi, \psi \phi / \psi \in \mathbf{L}$.

A substructural logic $\mathbf{L}$ is called integral, if for every $\phi, \phi \backslash(1 \wedge \phi) \in \mathbf{L}$; it is called contractive, if for every $\phi, \phi \backslash \phi^{2} \in \mathbf{L}$; finally, it is called commutative, if for every $\phi, \psi, \phi \psi \backslash \psi \phi \in \mathbf{L}$. If a logic is $\mathbf{L}$ integral and additionally $0 \backslash \phi \in \mathbf{L}$, for every $\phi$, we say that $\mathbf{L}$ has weakening. It is easy to see that a logic is integral, contractive, commutative or has weakening, iff it includes the logic $\mathbf{F L}_{\mathbf{i}}, \mathbf{F L}_{\mathbf{c}}, \mathbf{F L}_{\mathbf{e}}$ or $\mathbf{F L}_{\mathbf{w}}$, respectively; the reader is referred to [11] for the definition of these four logics.

For every class $\mathcal{K}$ of pointed residuated lattices and for every set $\Phi$ of formulas over $\mathcal{L}$, let $\mathbf{L}(\mathcal{K})=\left\{\phi \in F m_{\mathcal{L}} \mid \mathcal{K} \models 1 \leq \phi\right\}$ and $\mathcal{V}(\Phi)=\operatorname{Mod}(\{1 \leq$ $\phi \mid \phi \in \Phi\})$. Moreover, if $\Sigma$ is a set of formulas over $L$ and $E$ is a set of equations over $L$, we define the set of equations $E q(\Sigma)=\{1 \leq \phi \mid \phi \in \Sigma\}$, and the set of formulas $F m(E)=\{t \backslash s \wedge s \backslash t \mid(t \approx s) \in E\}$. Let $s \approx t$ and $s_{i} \approx t_{i}, i \in I$, be equations in the language of $\mathcal{F} \mathcal{L}, \bar{x}$ the sequence of variables in them and $\mathcal{K}$ a subclass of $\mathcal{F} \mathcal{L}$. Following [2], we say that $s \approx t$ is a $\mathcal{K}$-consequence of $E=\left\{s_{i} \approx t_{i} \mid i \in I\right\}$, in symbols $E \models_{\mathcal{K}} s \approx t$, iff, for all $\mathbf{A} \in \mathcal{K}$ and every assignment $\bar{a}$ in $\mathbf{A}$ for the variables $\bar{x}$, if $\mathbf{A} \models s_{i}(\bar{a})=t_{i}(\bar{a})$, for all $i \in I$, then $\mathbf{A} \models s(\bar{a})=t(\bar{a})$. Note that if $E$ is finite, then

$$
E \models \mathcal{K} s \approx t \text { iff } \mathcal{K}=(\forall \bar{x})\left(\bigwedge_{i \in I} s_{i}(\bar{x})=t_{i}(\bar{x}) \Rightarrow s(\bar{x})=t(\bar{x})\right) .
$$


Theorem 2.5. [11]

(1) For every $\mathcal{K} \subseteq \mathcal{F} \mathcal{L}, \mathbf{L}(\mathcal{K})$ is a substructural logic and for every $\Phi \subseteq$ $F m_{\mathcal{L}}, \mathcal{V}(\Phi)$ is a subvariety of $\mathcal{F} \mathcal{L}$.

(2) The maps $\mathbf{L}: \mathbf{S}(\mathcal{F} \mathcal{L}) \rightarrow \mathbf{S L}$ and $\mathcal{V}: \mathbf{S L} \rightarrow \mathbf{S}(\mathcal{F} \mathcal{L})$ are mutually inverse, dual lattice isomorphisms.

(3) If a substructural logic $\mathbf{L}$ is axiomatized by a set of formulas $\Phi$, then the variety $\mathcal{V}(\mathbf{L})$ is axiomatized by the set of equations $E q(\Phi)$.

(4) If a subvariety $\mathcal{V}$ of $\mathcal{F} \mathcal{L}$ is axiomatized by a set of equations $E$, then the substructural logic $\mathbf{L}(\mathcal{V})$ is axiomatized by the set of formulas $F m(E)$.

(5) A substructural logic is commutative, integral or contractive iff the corresponding variety is.

(6) If $\Sigma \cup\{\phi\}$ is a subset of $F m_{\mathcal{L}}$ and $\mathbf{L}$ is a substructural logic, then

$$
\begin{gathered}
\Sigma \vdash_{\mathbf{L}} \phi \text { iff } E q(\Sigma) \models_{\mathcal{V}(\mathbf{L})} 1 \leq \phi, \text { and } \\
\phi \vdash_{\mathbf{L}} 1 \backslash \phi \wedge \phi \backslash 1
\end{gathered}
$$

(7) If $E \cup\{t \approx s\}$ is a set of equations in $\mathcal{L}$ and $\mathcal{V}$ is a subvariety of $\mathcal{F} \mathcal{L}$, then

$$
\begin{gathered}
E \models \mathcal{V} t \approx s \text { iff } F m(E) \vdash_{\mathbf{L}(\mathcal{V})} t \backslash s \wedge s \backslash t, \text { and } \\
s \approx t=\models \mathcal{V} s \backslash t \wedge t \backslash s \approx 1
\end{gathered}
$$

The theorem implies, in the terminology of [2], that every subvariety $\mathcal{V}$ of $\mathcal{F} \mathcal{L}$ is an equivalent algebraic semantics for the substructural $\operatorname{logic} \mathbf{L}(\mathcal{V})$, where the defining equation is $1 \approx \phi \wedge 1$ and the equivalence formula is $\phi \backslash \psi \wedge \psi \backslash \phi$.

Theorem 2.5 establishes a link between substructural logics and varieties of pointed residuated lattices and it allows for the interchange of algebraic and logical terminology throughout the paper. We will use the language of logic and algebra interchangingly throughout the paper, without explicit reference to the algebraization result.

An iterated conjugate is a composition of polynomials of the form $\lambda_{a}(x)=$ $a \backslash x a \wedge 1$ and $\rho_{b}(x)=b x / b \wedge 1$, for various values of $a$ and $b$. For example, $\gamma(x)=a \backslash(b \backslash(c x / c \wedge 1) b \wedge 1) a \wedge 1$ is an iterated conjugate. See [3] and [11] for further discussion on conjugate terms.

The following theorem is a weak version of the classical deduction theorem and is called parametrized local deduction theorem in [7].

Theorem 2.6. [11] If $\Sigma \cup \Delta \cup\{\phi\}$ is a subset of $F m_{\mathcal{L}}$ and $\mathbf{L}$ is a substructural logic, then

$$
\Sigma, \Delta \vdash_{\mathbf{L}} \phi \quad \text { iff } \quad \Sigma \vdash_{\mathbf{L}}\left(\prod_{i=1}^{n} \gamma_{i}\left(\psi_{i}\right)\right) \backslash \phi,
$$

for some non-negative integer $n$, iterated conjugates $\gamma_{i}$ and $\psi_{i} \in \Delta, i<n$.

In particular, when $\mathbf{L}$ is commutative, then

$$
\Sigma, \Delta \vdash_{\mathbf{L}} \phi \quad \text { iff } \quad \Sigma \vdash_{\mathbf{L}}\left(\prod_{i=1}^{n}\left(\psi_{i} \wedge 1\right)\right) \rightarrow \phi,
$$


for some non-negative integer $n$, and $\psi_{i} \in \Delta, i<n$.

We denote by $\Sigma_{\mathcal{L}}$ the set of all substitutions in the language $\mathcal{L}$ of (pointed) residuated lattices. Also, if $\Phi$ is a set of formulas, we set $\Sigma_{\mathcal{L}}(\Phi)=\{\sigma(\phi) \mid \sigma \in$ $\left.\Sigma_{\mathcal{L}}, \phi \in \Phi\right\}$. If $\mathbf{L}$ is a substructural logic and $\Phi$ is a set of formulas, we denote by $\mathbf{L}+\Phi$ the least substructural logic that contains $\mathbf{L} \cup \Phi$.

The following corollary is an easy consequence of Theorem 2.4 and Theorem 2.6.

Corollary 2.7. If $\Phi \cup\{\psi\}$ is a set of formulas, then $\mathbf{F L}+\Phi \vdash_{\mathbf{F L}} \psi$ iff $\Sigma_{\mathcal{L}}(\Phi) \vdash_{\text {FL }} \psi$.

The following lemma allows for a certain degree of commutativity when dealing with inequalities, the right-hand side of which is negated. Note that we cannot use Lemma 2.3 , as we do not assume cyclicity.

Lemma 2.8. Let $\phi_{i}$ and $\psi$ be formulas, and $\gamma_{i}$ iterated conjugates, for all $i \in\{1,2, \ldots, n\}$, where $n$ is a non-negative integer. If $\prod_{i=1}^{n} \gamma_{i}\left(\phi_{i}\right) \leq$ $-\psi$, then there exist iterated conjugates $\gamma_{i}^{\prime}$, for $i \in\{1,2, \ldots, n\}$, such that $\prod_{i=n}^{1}-\sim \gamma_{i}^{\prime}\left(\phi_{i}\right) \leq-\psi$. [Note the change in the order of the product.]

Proof. If $\gamma_{1}\left(\phi_{1}\right) \gamma_{2}\left(\phi_{2}\right) \gamma_{3}\left(\phi_{3}\right) \cdots \gamma_{n}\left(\phi_{n}\right) \leq-\psi$, then we have

$$
\left[-\sim \gamma_{1}\left(\phi_{1}\right)\right] \gamma_{2}\left(\phi_{2}\right) \gamma_{3}\left(\phi_{3}\right) \cdots \gamma_{n}\left(\phi_{n}\right) \leq-\psi,
$$

by Lemma 2.2(13). Recalling that $\rho_{a}(b) a \leq a b$, by Lemma 2.1, we have successively

$$
\begin{gathered}
\rho_{\left[-\sim \gamma_{1}\left(\phi_{1}\right)\right]}\left(\gamma_{2}\left(\phi_{2}\right)\right)\left[-\sim \gamma_{1}\left(\phi_{1}\right)\right] \gamma_{3}\left(\phi_{3}\right) \cdots \gamma_{n}\left(\phi_{n}\right) \leq-\psi, \\
\rho_{\left[-\sim \gamma_{1}\left(\phi_{1}\right)\right]}\left(\gamma_{2}\left(\phi_{2}\right)\right) \rho_{\left[-\sim \gamma_{1}\left(\phi_{1}\right)\right]}\left(\gamma_{3}\left(\phi_{3}\right)\right)\left[-\sim \gamma_{1}\left(\phi_{1}\right)\right] \cdots \gamma_{n}\left(\phi_{n}\right) \leq-\psi,
\end{gathered}
$$

and finally, for $a=\left[-\sim \gamma_{1}\left(\phi_{1}\right)\right]$,

$$
\rho_{a}\left(\gamma_{2}\left(\phi_{2}\right)\right) \rho_{a}\left(\gamma_{3}\left(\phi_{3}\right)\right) \cdots \rho_{a}\left(\gamma_{n}\left(\phi_{n}\right)\right)\left[-\sim \gamma_{1}\left(\phi_{1}\right)\right] \leq-\psi
$$

or simply, for $\gamma_{i}^{\prime}\left(\phi_{i}\right)=\rho_{a}\left(\gamma_{i}\left(\phi_{i}\right)\right)$,

$$
\gamma_{2}^{\prime}\left(\phi_{2}\right) \gamma_{3}^{\prime}\left(\phi_{3}\right) \cdots \gamma_{n}^{\prime}\left(\phi_{n}\right)\left[-\sim \gamma_{1}\left(\phi_{1}\right)\right] \leq-\psi .
$$

By another application of Lemma 2.2(13), we have

$$
\left[-\sim \gamma_{2}^{\prime}\left(\phi_{2}\right)\right] \gamma_{3}^{\prime}\left(\phi_{3}\right) \cdots \gamma_{n}^{\prime}\left(\phi_{n}\right)\left[-\sim \gamma_{1}\left(\phi_{1}\right)\right] \leq-\psi
$$

and proceeding in the same spirit as above, we have, for $\gamma_{i}^{\prime \prime}\left(\phi_{i}\right)=\rho_{b}\left(\gamma_{i}^{\prime}\left(\phi_{i}\right)\right)$ and $b=\left[-\sim \gamma_{2}^{\prime}\left(\phi_{2}\right)\right]$,

$$
\gamma_{3}^{\prime \prime}\left(\phi_{3}\right) \cdots \gamma_{n}^{\prime \prime}\left(\phi_{n}\right)\left[-\sim \gamma_{2}^{\prime}\left(\phi_{2}\right)\right]\left[-\sim \gamma_{1}\left(\phi_{1}\right)\right] \leq-\psi .
$$

Proceeding inductively, we obtain

$$
\left[-\sim \bar{\gamma}_{n}\left(\phi_{n}\right)\right] \cdots\left[-\sim \bar{\gamma}_{2}\left(\phi_{2}\right)\right]\left[-\sim \bar{\gamma}_{1}\left(\phi_{1}\right)\right] \leq-\psi,
$$

for some iterated conjugates $\bar{\gamma}_{i}$. 


\section{Glivenko equivalence}

We introduce the notion of Glivenko equivalence between subvarieties of $\mathcal{F} \mathcal{L}$. Via the algebraization theorem, Theorem 2.5, there is an associated relation between substructural logics, for which we will use the same name. Glivenko equivalence will serve as a unifying concept that will connect the different Glivenko properties we will be consider.

Lemma 3.1. Let $\mathcal{W}, \mathcal{V}$ be subvarieties of $\mathcal{F} \mathcal{L}$ and let $r, s, t, s_{i}, t_{i}$ terms over $\mathcal{F} \mathcal{L}$, for $i \in I$. We consider the sets $E=\left\{s_{i} \approx t_{i} \mid i \in I\right\}, D=\left\{1 \leq t_{i} \mid i \in I\right\}$, $\sim E=\left\{\sim s_{i} \approx \sim t_{i} \mid i \in I\right\}$ and $\sim D=\left\{1 \leq \sim t_{i} \mid i \in I\right\}$. The following statements are equivalent.

(1) $\mathcal{V}=\sim s \approx \sim t$ iff $\mathcal{W}=\sim s \approx \sim t$.

(2) $\mathcal{V}=-s \approx-t$ iff $\mathcal{W}=-s \approx-t$.

(3) $\mathcal{V}=\sim s \leq \sim t$ iff $\mathcal{W} \models \sim s \leq \sim t$.

(4) $\mathcal{V}=r \leq \sim t$ iff $\mathcal{W}=r \leq \sim t$.

(5) $\mathcal{V} \mid=1 \leq \sim t$ iff $\mathcal{W}=1 \leq \sim t$.

(6) $D \mid=\mathcal{V} 1 \leq \sim t$ iff $D \models \mathcal{W} 1 \leq \sim t$.

(7) $\sim D \mid=\mathcal{V} 1 \leq \sim t$ iff $\sim D \mid \models_{\mathcal{W}} 1 \leq \sim t$.

(8) $E \models \mathcal{V} \sim s \approx \sim t$ iff $E \models \mathcal{W} \sim s \approx \sim t$.

(9) $\sim E \models \mathcal{V} \sim s \approx \sim t$ iff $\sim E||_{\mathcal{W}} \sim s \approx \sim t$.

Proof. Assume that $(1)$ holds. Then, $\mathcal{V}$ satisfies $-s \approx-t$ iff it satisfies $\sim-s \approx \sim-t$, by Lemma 2.2(6). By (1), this is true iff $\mathcal{W}$ satisfies $\sim-s \approx$ $\sim-t$; i.e., iff $\mathcal{W}$ satisfies $-s \approx-t$. Therefore, (1) implies (2); the converse is obtained by interchanging the two negation operations.

Note that $\sim s \leq \sim t$ iff $\sim s \approx \sim(s \vee t)$, by Lemma 2.2(1); also $\sim s \approx \sim t$ is equivalent to the conjunction of $\sim s \leq \sim t$ and $\sim t \leq \sim s$. The equivalence between (1) and (3) follows from these two facts.

Obviously, (4) implies both (3) and (5). (5) implies (4), since $r \leq \sim t$ iff $1 \leq r \backslash \sim t$ iff $1 \leq \sim(t r)$, by Lemma 2.2(8), and (3) implies (4), since $r \leq \sim t$ iff $\sim-r \leq \sim t$, by Lemma 2.2(2,3,4). Consequently, (1)-(5) are all equivalent. Moreover, the same argument shows that (9) implies (7). It is clear that (8) implies (9) and that (7) implies (5). We will show that (5) implies (6) and that (6) implies (8).

Assume that (5) holds. We have $\left\{1 \leq t_{i} \mid i \in I\right\} \mid=\mathcal{V} 1 \leq \sim t$ iff there exists a natural number $n$ and iterated conjugates $\gamma_{k}$ over a sequence of terms, such that $\models_{\mathcal{V}} 1 \leq \prod_{k=1}^{n} \gamma_{k}\left(t_{i_{k}}\right) \backslash \sim t=\sim\left[t \cdot \prod_{k=1}^{n} \gamma_{k}\left(t_{i_{k}}\right)\right]$, by using Theorem 2.5(6) and Theorem 2.6. By (5) the same equation holds in $\mathcal{W}$ for the same $n$ and the same iterated conjugates, hence $\left\{1 \leq t_{i} \mid i \in I\right\} \models_{\mathcal{W}} 1 \leq \sim t$; consequently, (6) holds.

Assume, now, that (6) holds. We have $\sim s \approx \sim t$ iff $\sim s \leq \sim t$ and $\sim t \leq \sim s$, iff $1 \leq \sim s \backslash \sim t=\sim(t \cdot \sim s)$ and $1 \leq \sim(s \cdot \sim t)$, by Lemma 2.2(8), iff $1 \leq \sim(t \cdot \sim s) \wedge \sim(s \cdot \sim t)$, iff $1 \leq \sim p$, where $p=(t \cdot \sim s) \vee(s \cdot \sim t)$, by Lemma 2.2(1). Moreover, $s \approx t$ iff $1 \leq s \backslash t \wedge t \backslash s$. So, (8) can be written in a form that is a special case of (6). 
If any of the equivalent statements of the previous lemma holds, we say that the variety $\mathcal{V}$ is Glivenko equivalent to the variety $\mathcal{W}$. Glivenko equivalence coincides with the notion of negative equivalence of S. Odintsov, for the special cases considerd in [15]. Obviously, Glivenko equivalence is an equivalence relation on $\mathbf{S}(\mathcal{F} \mathcal{L})$. It is clear that if $\mathcal{V}, \mathcal{W}$ are Glivenko equivalent and $\mathcal{V} \subseteq \mathcal{U} \subseteq \mathcal{W}$, then $\mathcal{U}$ is Glivenko equivalent to $\mathcal{V}$ and $\mathcal{W}$. So, the equivalence classes of the Glivenko equivalence relation are convex.

We say that the substructural logics $\mathbf{K}$ and $\mathbf{L}$ are Glivenko equivalent, if for all formulas $\phi$,

$$
\vdash_{\mathbf{K}} \sim \phi \text { iff } \vdash_{\mathbf{L}} \sim \phi
$$

By Lemma 3.1, $\mathbf{K}$ and $\mathbf{L}$ are Glivenko equivalent iff $\mathcal{V}(\mathbf{K})$ and $\mathcal{V}(\mathbf{L})$ are Glivenko equivalent. It follows from Lemma 3.1 that $\sim$ can be replaced by - in the above definition.

For every variety $\mathcal{V}$ of pointed residuated lattices, let $\mathbf{G}(\mathcal{V})$ be the subvariety of $\mathcal{F} \mathcal{L}$ axiomatized by the equations $\sim s \approx \sim t$, where $s, t$ range over all pairs of terms such that the equation $s \approx t$ holds in $\mathcal{V}$. The variety $\mathbf{G}(\mathcal{V})$ is called the Glivenko variety of $\mathcal{V}$.

Lemma 3.2. For every subvariety $\mathcal{V}$ of $\mathcal{F} \mathcal{L}$, the variety $\mathbf{G}(\mathcal{V})$ is also axiomatized by the equations $-s \approx-t$, where $s \approx t$ holds in $\mathcal{V}$.

Proof. Consider the variety $\mathbf{G}^{\prime}(\mathcal{V})$ axiomatized by the equations $-s \approx-t$, where $s \approx t$ holds in $\mathcal{V}$; we will show that $\mathbf{G}^{\prime}(\mathcal{V})=\mathbf{G}(\mathcal{V})$. For every equation $s \approx t$ valid in $\mathcal{V}$, the equation $-s \approx-t$ is valid in $\mathcal{V}$, as well. So, $\mathbf{G}(\mathcal{V})$ satisfies the equation $\sim-s \approx \sim-t$, hence it also satisfies the equation $-\sim-s \approx-\sim-t$. In view of Lemma 2.2(4), we have that $-s \approx-t$ holds in $\mathbf{G}(\mathcal{V})$. Thus, $\mathbf{G}(\mathcal{V}) \subseteq \mathbf{G}^{\prime}(\mathcal{V})$. Likewise, we obtain the converse inclusion.

Lemma 3.3. Let $\mathcal{U}, \mathcal{V}, \mathcal{W}$ be subvarieties of $\mathcal{F} \mathcal{L}$.

(1) $\mathbf{G}$ is a closure operator on $\mathbf{S}(\mathcal{F} \mathcal{L})$; i.e.

(a) $\mathcal{V} \subseteq \mathbf{G}(\mathcal{V})$

(b) if $\mathcal{V} \subseteq \mathcal{W}$, then $\mathbf{G}(\mathcal{V}) \subseteq \mathbf{G}(\mathcal{W})$, and

(c) $\mathbf{G}(\mathbf{G}(\mathcal{V}))=\mathbf{G}(\mathcal{V})$.

(2) The varieties $\mathcal{V}$ and $\mathbf{G}(\mathcal{V})$ are Glivenko equivalent.

(3) The varieties $\mathcal{V}$ and $\mathcal{W}$ are Glivenko equivalent iff $\mathbf{G}(\mathcal{V})=\mathbf{G}(\mathcal{W})$.

(4) The variety $\mathbf{G}(\mathcal{V})$ is the largest subvariety of $\mathcal{F} \mathcal{L}$ that is Glivenko equivalent to $\mathcal{V}$.

Proof. (1) For (a), note that if $\sim s \approx \sim t$ is an axiom of $\mathbf{G}(\mathcal{V})$, namely $s \approx t$ is valid in $\mathcal{V}$, then $\sim s \approx \sim t$ is valid in $\mathcal{V}$. Thus, $\mathcal{V}$ is a subvariety of $\mathbf{G}(\mathcal{V})$. The fact that $\mathbf{G}$ is increasing is clear from its definition. To show that $\mathbf{G}(\mathbf{G}(\mathcal{V})) \subseteq \mathbf{G}(\mathcal{V})$, let $\sim s \approx \sim t$ be an axiom of $\mathbf{G}(\mathcal{V})$. Then, $-\sim s \approx-\sim t$ holds in $\mathbf{G}(\mathcal{V})$, hence $\sim-\sim s \approx \sim-\sim t$ holds in $\mathbf{G}(\mathbf{G}(\mathcal{V}))$. Thus, $\mathbf{G}(\mathbf{G}(\mathcal{V}))$ satisfies $\sim s \approx \sim t$, by Lemma $2.2(4)$. 
(2) If $\mathcal{V}$ satisfies $-s \approx-t$, then $\mathbf{G}(\mathcal{V})$ satisfies $\sim-s \approx \sim-t$, hence it satisfies $-s \approx-t$, by Lemma 2.2(6). Conversely, if $-s \approx-t$ holds in $\mathbf{G}(\mathcal{V})$, then it also holds in $\mathcal{V}$, by (1a). Thus, $\mathcal{V}$ and $\mathbf{G}(\mathcal{V})$ are Glivenko equivalent.

(3) If $\mathcal{V}$ and $\mathcal{W}$ are Glivenko equivalent, then $\mathbf{G}(\mathcal{V})$ and $\mathbf{G}(\mathcal{W})$ are Glivenko equivalent, by (2). Thus, if $\sim s \approx \sim t$ is an axiom of $\mathbf{G}(\mathcal{V})$, then it is valid in $\mathbf{G}(\mathcal{W})$. So, $\mathbf{G}(\mathcal{W}) \subseteq \mathbf{G}(\mathcal{V})$. The other inclusion is obtained in a similar way, hence $\mathbf{G}(\mathcal{V})=\mathbf{G}(\mathcal{W})$. Conversely, if $\mathbf{G}(\mathcal{V})=\mathbf{G}(\mathcal{W})$, then $\mathcal{V}$, $\mathcal{W}$ are Glivenko equivalent, by $(2)$.

(4) If $\mathcal{V}, \mathcal{W}$ are Glivenko equivalent, then $\mathbf{G}(\mathcal{V})=\mathbf{G}(\mathcal{W})$, by (3). Since $\mathcal{W} \subseteq \mathbf{G}(\mathcal{W})$, by $(1)$, we have $\mathcal{W} \subseteq \mathbf{G}(\mathcal{V})$. So, in view of $(2), \mathbf{G}(\mathcal{V})$ is the largest subvariety of $\mathcal{F} \mathcal{L}$ that is Glivenko equivalent to $\mathcal{V}$.

For a substructural logic $\mathbf{L}$ we define the Glivenko logic of $\mathbf{L}$ to be $\mathbf{G}(\mathbf{L})=$ $\mathbf{L}(\mathbf{G}(\mathcal{V}(\mathbf{L})))$. It follows from the preceding theorem and from Theorem 2.5 that $\mathbf{G}(\mathbf{L})$ is the smallest substructural logic that is Glivenko equivalent to L.

By definition $\mathbf{G}(\mathcal{V}(\mathbf{L}))$ is axiomatized by the equations $\sim s \approx \sim t$, where $s \approx t$ ranges over all equations valid in $\mathcal{V}(\mathbf{L})$. Recalling that $s \approx t$ is valid in $\mathcal{V}(\mathbf{L})$ iff the formula $s \backslash t \wedge t \backslash s$ is in $\mathbf{L}$ iff both of $s \backslash t$ and $t \backslash s$ are in $\mathbf{L}$ we have that $\mathbf{G}(\mathbf{L})$ is axiomatized by the formulas $\sim s \backslash \sim t \wedge \sim t \backslash \sim s$, where $s \backslash t$ and $t \backslash s$ are in $\mathbf{L}$. Therefore, $\mathbf{G}(\mathbf{L})=\mathbf{F L}+\{\sim \psi \backslash \sim \phi \mid \phi \backslash \psi \in \mathbf{L}\}$. We provide an alternative axiomatization for $\mathbf{G}(\mathbf{L})$.

Proposition 3.4. If $\mathbf{L}$ is a substructural logic, then $\mathbf{G}(\mathbf{L})$ is axiomatized relative to $\mathbf{F L}$ by either one of the sets $\{-\sim \phi \mid \phi \in \mathbf{L}\}$ and $\{\sim-\phi \mid \phi \in \mathbf{L}\}$; i.e., $\mathbf{G}(\mathbf{L})=\mathbf{F L}+\{-\sim \phi \mid \phi \in \mathbf{L}\}=\mathbf{F L}+\{\sim-\phi \mid \phi \in \mathbf{L}\}$.

Proof. We will show that $\mathbf{G}(\mathbf{L})=\mathbf{M}$, where $\mathbf{M}=\mathbf{F L}+\{-\sim \phi \mid \phi \in \mathbf{L}\}$.

If $-\sim \phi$ is an axiom of $\mathbf{M}$ for $\phi \in \mathbf{L}$, then $1 \backslash \phi \in \mathbf{L}$; hence

$$
-\sim \phi=\sim \phi \backslash 0=\sim \phi \backslash \sim 1 \in \mathbf{G}(\mathbf{L}) .
$$

Consequently, $\mathbf{M} \subseteq \mathbf{G}(\mathbf{L})$.

Conversely, suppose that $\sim \psi \backslash \sim \phi$ is an axiom of $\mathbf{G}(\mathbf{L})$ for $\phi \backslash \psi \in \mathbf{L}$. So, $-\sim(\phi \backslash \psi) \in \mathbf{M}$ and $\rho_{\phi}(-\sim(\phi \backslash \psi)) \in \mathbf{M}$.

By Lemma 2.1(4), we have $(0 / \psi) \phi(\phi \backslash \psi) \leq 0=\sim 1$, so

$$
(0 / \psi) \phi[-\sim(\phi \backslash \psi)] \leq 0,
$$

by Lemma $2.2(13)$. Hence, $\phi[-\sim(\phi \backslash \psi)] \leq(0 / \psi) \backslash 0=(\sim \psi) \backslash 0$. Since

$$
\rho_{\phi}(-\sim(\phi \backslash \psi)) \phi=[\phi(-\sim(\phi \backslash \psi)) / \phi \wedge 1] \phi \leq \phi(-\sim(\phi \backslash \psi)),
$$

we have $\rho_{\phi}(-\sim(\phi \backslash \psi)) \phi \leq(\sim \psi) \backslash 0$. So,

$$
\rho_{\phi}(-\sim(\phi \backslash \psi)) \leq((\sim \psi) \backslash 0) / \phi=(\sim \psi) \backslash(\sim \phi),
$$

by Lemma 2.1(7). By $\left(\mathrm{mp}_{\ell}\right)$ of Theorem 2.4 , we have $(\sim \psi) \backslash(\sim \phi) \in \mathbf{M}$. Consequently, $\mathbf{G}(\mathbf{L}) \subseteq \mathbf{M}$. 
An axiomatization of $\mathbf{G}(\mathbf{L})$ is also given in [15], for the special case of extensions of Johansson's logic.

We know form Theorem 3.3(4) that given a logic $\mathbf{L}$ there exists a smallest logic $\mathbf{G}(\mathbf{L})$ that is Glivenko equivalent to $\mathbf{L}$. For every substructural logic $\mathbf{L}$, we define the logic

$$
\mathbf{M}(\mathbf{L})=\mathbf{F L}+\{\phi \mid-\sim \gamma(\phi) \in \mathbf{L}, \text { for every } \gamma \in \Gamma\}
$$

where $\Gamma$ denotes the set of all iterated conjugates.

Theorem 3.5. For every substructural logic $\mathbf{L}$, the logic $\mathbf{M}(\mathbf{L})$ is the greatest element of the Glivenko equivalence class of $\mathbf{L}$.

Proof. If $\psi \in \mathbf{L}$, then $\gamma(\psi) \in \mathbf{L}$, by (pn) and (adju) of Theorem 2.4, and $-\sim \gamma(\psi) \in \mathbf{L}$, by Lemma $2.2(3)$ and $\left(\mathrm{mp}_{\ell}\right)$ of Theorem 2.4 ; hence $\psi \in \mathbf{M}(\mathbf{L})$. Therefore, $\mathbf{L} \subseteq \mathbf{M}(\mathbf{L})$.

If $-\psi \in \mathbf{M}(\mathbf{L})$, then $\mathbf{F L}+\Phi \vdash_{\mathbf{F L}}-\psi$, where

$$
\Phi=\{\phi \mid-\sim \gamma(\phi) \in \mathbf{L}, \text { for every } \gamma \in \Gamma\} .
$$

By Corollary 2.7, we have $\Sigma_{\mathcal{L}}(\Phi) \vdash_{\mathbf{F L}}-\psi$. We will show that $\Sigma_{\mathcal{L}}(\Phi)=\Phi$.

Assume that $\phi \in \Phi, \sigma \in \Sigma_{\mathcal{L}}$ and $\gamma \in \Gamma$. Let $\gamma^{\prime}$ be the iterated conjugate obtained from $\gamma$ by replacing all common variables $x_{i}$ of $\gamma$ and $\phi$ in $\gamma$ by new variables $y_{i}$ not appearing in $\gamma$ or $\phi$. Also, let $\sigma^{\prime}$ be the substitution that maps the variables $y_{i}$ to the variables $x_{i}$ and otherwise behaves like $\sigma$. It is easy to see that $\sigma^{\prime}\left(-\sim \gamma^{\prime}(\phi)\right)=-\sim \gamma(\sigma(\phi))$. Since $\phi \in \Phi$, we have $-\sim \gamma^{\prime}(\phi) \in L$ and hence $-\sim \gamma(\sigma(\phi))=\sigma^{\prime}\left(-\sim \gamma^{\prime}(\phi)\right) \in L$. Thus, $\sigma(\phi) \in \Phi$.

Consequently, $\Phi \vdash_{\mathbf{F L}}-\psi$, so there are $\phi_{i} \in \Phi$ and iterated conjugates $\gamma_{i}$, for $i \in\{1,2, \ldots, n\}$, for some non-negative integer $n$, such that

$$
\prod_{i=1}^{n} \gamma_{i}\left(\phi_{i}\right) \leq-\psi
$$

By Lemma 2.8, we have

$$
\prod_{i=n}^{1}-\sim \gamma_{i}^{\prime}\left(\phi_{i}\right) \leq-\psi
$$

Since $\phi_{i} \in \Phi$, for all $i$, we have that $-\sim \gamma_{i}^{\prime}\left(\phi_{i}\right) \in \mathbf{L}$, for all $i$; hence $\prod_{i=n}^{1}-\sim \gamma_{i}^{\prime}\left(\phi_{i}\right) \in \mathbf{L}$, by (pn) of Theorem 2.4 and $-\psi \in \mathbf{L}$, by $\left(\mathrm{mp}_{\ell}\right)$ of the same theorem. Consequently, $\mathbf{L}$ and $\mathbf{M}(\mathbf{L})$ are Glivenko equivalent.

Now, assume that $\mathbf{K}$ is a substructural logic that is Glivenko equivalent to $\mathbf{L}$. If $\psi \in \mathbf{K}$, then, by Theorem 2.4, we have that $\sigma(\psi) \in \mathbf{K}$, for every substitution $\sigma, \gamma(\sigma(\psi)) \in \mathbf{K}$, for every iterated conjugate $\gamma$, and $-\sim \gamma(\sigma(\psi)) \in \mathbf{K}$. By the Glivenko equivalence, $-\sim \gamma(\sigma(\psi)) \in \mathbf{L}$, for all $\sigma \in \Sigma_{\mathcal{L}}$ and for all $\gamma \in \Gamma$; thus $\psi \in \mathbf{M}(\mathbf{L})$. Consequently, $\mathbf{K}$ is contained in $\mathbf{M}(\mathbf{L})$.

A similar result is shown in [15] for the special case considered in the paper. 
The definitions and results in this as well as in the following sections can be transferred from subvarieties of $\mathcal{F} \mathcal{L}$ to substructural logics over $\mathbf{F L}$ and vice versa. For example, for a subvariety $\mathcal{V}$ of $\mathcal{F} \mathcal{L}$ we define $\mathbf{M}(\mathcal{V})=$ $\mathcal{V}(\mathbf{M}(\mathbf{L}(\mathcal{V})))$.

It follows, by Lemma 3.3(4) and Theorem 3.5, that the Glivenko equivalence classes are intervals in $\mathbf{S}(\mathcal{F} \mathcal{L})$ of the form $[\mathbf{M}(\mathcal{V}), \mathbf{G}(\mathcal{V})]$. Also, the classes of the Glivenko equivalence between logics are intervals of the form $[\mathbf{G}(\mathbf{L}), \mathbf{M}(\mathbf{L})]$.

\section{Glivenko properties}

In this section we discuss when a Glivenko property holds for a substructural logic $\mathbf{K}$ relative to a substructural logic $\mathbf{L}$. As mentioned in the introduction, we consider three types of Glivenko properties. We provide a characterization for each of them in terms of different types of involutiveness that we introduce below.

A substructural logic $\mathbf{L}$ is called left involutive, if $\vdash_{\mathbf{L}} \sim-\phi \backslash \phi$, for every $\phi$. We say that $\mathbf{L}$ is left weakly involutive, if $\sim-\phi \vdash_{\mathbf{L}} \phi$, for every $\phi$, and that it is left Glivenko involutive, if $\vdash_{\mathbf{L}} \sim-\phi$ implies $\vdash_{\mathbf{L}} \phi$, for every $\phi$. Clearly, left involutiveness is the strongest and left Glivenko involutiveness is the weakest among the three properties. In Section 4.3 - see Propositions 4.9 and 4.10 - we will see that the associated implications are strict.

4.1. The Glivenko property. We say that the left Glivenko property holds for $\mathbf{K}$ relative to $\mathbf{L}$, or that $\mathbf{K}$ has the left Glivenko property relative to $\mathbf{L}$, if $\vdash_{\mathbf{L}} \phi$ iff $\vdash_{\mathbf{K}} \sim-\phi$, for all $\phi$. The opposite of the left Glivenko property (obtained by interchanging $\sim$ and - ) is the right Glivenko property and the conjunction of the two is the Glivenko property. We define the Glivenko property for subvarieties of $\mathcal{F} \mathcal{L}$ by referring to their corresponding substrctural logics. The following result then can be reformulated for subvarieties of $\mathcal{F} \mathcal{L}$ in the obvious way.

Proposition 4.1. If $\mathbf{L}$ and $\mathbf{K}$ are substructural logics, then the following are equivalent.

(1) The left Glivenko property holds for $\mathbf{K}$ relative to $\mathbf{L}$.

(2) $\mathbf{K}$ and $\mathbf{L}$ are Glivenko equivalent and $\mathbf{L}$ is left Glivenko involutive.

(3) $\mathbf{L}=\mathbf{M}(\mathbf{K})$ and $\mathbf{M}(\mathbf{K})$ is left Glivenko involutive.

Proof. We first establish the equivalence of (1) and (2). By setting $\sim \phi$ for $\phi$ in (1), it follows by Lemma 2.2(4) that $\mathbf{K}$ and $\mathbf{L}$ are Glivenko equivalent. In particular, $\vdash_{\mathbf{K}} \sim-\phi$ iff $\vdash_{\mathbf{L}} \sim-\phi$; hence $\vdash_{\mathbf{L}} \sim-\phi$ iff $\vdash_{\mathbf{L}} \phi$, for every $\phi$, by (1). Conversely, if (2) holds, then $\vdash_{\mathbf{K}} \sim-\phi$ iff $\vdash_{\mathbf{L}} \sim-\phi$ iff $\vdash_{\mathbf{L}} \phi$, by the assumption that $\mathbf{L}$ is left Glivenko involutive.

Obviously, (3) implies (2). For the converse implication, note that $\mathbf{L} \subseteq$ $\mathbf{M}(\mathbf{K})$, since $\mathbf{K}$ and $\mathbf{L}$ are Glivenko equivalent. Moreover, if $\vdash_{\mathbf{M}(\mathbf{K})} \phi$, then 
$\vdash_{\mathbf{M}(\mathbf{K})} \sim-\phi$. By Glivenko equivalence, we have $\vdash_{\mathbf{L}} \sim-\phi$, so $\vdash_{\mathbf{L}} \phi$, since $\mathbf{L}$ is left Glivenko involutive. Thus, $\mathbf{M}(\mathbf{K}) \subseteq \mathbf{L}$.

It follows from Proposition 4.1 that in every Glivenko equivalence class $[\mathbf{G}(\mathbf{K}), \mathbf{M}(\mathbf{K})]$ there is at most one left Glivenko involutive logic and it is equal to $\mathbf{M}(\mathbf{K})$, when it exists. The corresponding statement and the analogue of Proposition 4.1 hold for subvarieties of $\mathcal{F} \mathcal{L}$. Conditions on the existence of the left Glivenko involutive logic in a Glivenko equivalence class will be discussed in Section 5; see page 25 .

We show that there exists a substructural logic $\mathbf{K}$ for which the Glivenko property does not hold (relative to any logic $\mathbf{L}$ ). We will state and prove this result in the terminology of algebra; i.e. we will show that there is a subvariety $\mathcal{V}$ of $\mathcal{F} \mathcal{L}$, for which the Glivenko property does not hold. By Proposition 4.1, it is enough to show that $\mathbf{M}(\mathcal{V})$ is not left Glivenko involutive.

We define an order relation on the set $A=\{\perp, u, 1, \top\}$, by $\perp<u<$ $1<\top$. Moreover, we define an idempotent multiplication, for which $\perp$ is an absorbing and 1 a unit element, by $\top u=\top$ and $u \top=u$. It is easy to check that multiplication preserves order, hence it preserves arbitrary joins, as well, since $\mathbf{A}$ is totally ordered. Therefore, multiplication is residuated with respect to the order and it can be easily checked that it is associative. We denote by $\mathbf{A}$ the associated pointed residuated lattice, where $0=\perp$.

Proposition 4.2. The subvariety of $\mathcal{F} \mathcal{L}$ that is generated by $\mathbf{A}$ does not enjoy the left Glivenko property. Similarly, the variety generated by $\mathbf{A}^{o p}$ does not enjoy the right Glivenko property.

Proof. It is easy to see that $\mathbf{A}$ does not have any subalgebras or homomorphic images other than the trivial and the universal. Therefore, the variety $\mathcal{W}$ generated by $\mathbf{A}$ is an atom, see $[9]$, and $\mathbf{M}(\mathcal{W})=\mathcal{W}$. To show that $\mathcal{W}$ is not left Glivenko involutive, it suffices to show that there is a term $t$ such that $\mathbf{A} \models 1 \leq \sim-t$, but not $\mathbf{A} \models 1 \leq t$. Such a term is $t(x)=1 /[x \vee(x \backslash 1)]$. Indeed, it is not hard to verify that $t^{\mathbf{A}}(x)=u$ and $\sim-t^{\mathbf{A}}(x)=\top$, if $x \neq 1$, $t^{\mathbf{A}}(1)=1$ and $\sim-t^{\mathbf{A}}(1)=\top$.

On the other hand, we have the following result.

Proposition 4.3. $\mathbf{M}(\mathbf{K})$ is Glivenko involutive whenever $\mathbf{K}$ is a substructural logic that contains $\mathbf{F} \mathbf{L}_{\mathbf{e w}}$. Thus, the Glivenko property holds for every substructural logic over $\mathbf{F L}_{\mathbf{e w}}$.

Proof. It follows from Theorem 3.5, and the fact that conjugates do not contribute anything in the commutative integral case, that $\mathbf{M}(\mathbf{K})=\mathbf{F L}_{\text {ew }}+$ $\{\phi \mid \sim \sim \phi \in \mathbf{K}\}$. We will show that $\mathbf{M}(\mathbf{K})$ is Glivenko involutive. If $\vdash_{\mathbf{M}(\mathbf{K})} \sim \sim \phi$, then, by Theorem 2.6 and integrality, there exist $\phi_{i}, i \in I$, such that $\vdash_{\mathbf{K}} \sim \sim \phi_{i}$ and $\vdash_{\mathbf{F L}_{\mathbf{e w}}}\left(\prod \phi_{i}\right) \rightarrow \sim \sim \phi$. It follows from Lemma 2.8, 
commutativity and integrality that $\vdash_{\mathbf{F L}}\left(\Pi \sim \sim \phi_{i}\right) \rightarrow \sim \sim \phi$; alternatively, using terminology and results that have not been itroduced yet, it follows from the fact that $\lambda$ is a nucleus in the commutative case according to Lemma 5.2 of the next section. Therefore, $\vdash_{\mathbf{K}} \sim \sim \phi$, by $\left(\mathrm{mp}_{\ell}\right)$ of Theorem 2.4; hence $\vdash_{\mathbf{M}(\mathbf{K})} \phi$.

4.2. The deductive Glivenko property. We say that the left deductive Glivenko property holds for $\mathbf{K}$ relative to $\mathbf{L}$, if $\Sigma \vdash_{\mathbf{L}} \phi$ iff $\Sigma \vdash_{\mathbf{K}} \sim-\phi$, for all $\Sigma \cup\{\phi\}$. The right deductive Glivenko property is defined as the opposite statement to the left deductive Glivenko property; the deductive Glivenko property is the conjunction of the two properties.

Proposition 4.4. If $\mathbf{L}$ and $\mathbf{K}$ are substructural logics and $\Phi \cup\{\psi\}$ are formulas, then the following are equivalent.

(1) The left deductive Glivenko property holds for $\mathbf{K}$ relative to $\mathbf{L}$.

(2) $\Phi \vdash_{\mathbf{L}} \psi$ iff $\sim-\Phi \vdash_{\mathbf{K}} \sim-\psi$, for all $\Phi \cup\{\psi\}$.

(3) $\mathbf{K}$ and $\mathbf{L}$ are Glivenko equivalent and $\mathbf{L}$ is left weakly involutive.

(4) $\mathbf{L}=\mathbf{M}(\mathbf{K})$ and $\mathbf{M}(\mathbf{K})$ is left weakly involutive.

Proof. (1) $\Rightarrow(2)$. Assume that (1) holds and let $\sim-\Phi \vdash_{\mathbf{K}} \sim-\psi$, for some $\Phi \cup\{\psi\}$. Since $\phi \vdash_{\mathbf{K}} \sim-\phi$ for all $\phi \in \Phi$, we get $\Phi \vdash_{\mathbf{K}} \sim-\psi$, by the transitivity of $\vdash_{\mathbf{K}}$. By (1), we obtain $\Phi \vdash_{\mathbf{L}} \psi$. Conversely, let $\Phi \vdash_{\mathbf{L}} \psi$, for some $\Phi \cup\{\psi\}$. Taking $\{\sim-\chi\}$ for $\Sigma$ and $\chi$ for $\phi$ in (1), we obtain $\sim-\chi \vdash_{\mathbf{L}} \chi$, for every $\chi$. So, $\sim-\Phi \vdash_{\mathbf{L}} \phi$, for all $\phi \in \Phi$; hence $\sim-\Phi \vdash_{\mathbf{L}} \psi$, by transitivity. By $(1)$, we get $\sim-\Phi \vdash_{\mathbf{K}} \sim-\psi$.

$(2) \Rightarrow(3)$. Recall that $\sim-\sim-\psi=\sim-\psi$; so, for $\Phi=\{\sim-\psi\}$, (2) yields $\sim-\psi \vdash_{\mathbf{L}} \psi$. Moreover, by substituting the empty set for $\Phi$ and $\sim \psi$ for $\psi$ in (2), we obtain $\vdash_{\mathbf{L}} \sim \psi$ iff $\vdash_{\mathbf{K}} \sim \psi$, for all $\psi$, by Lemma 2.2(4). Consequently, $\mathbf{K}$ and $\mathbf{L}$ are Glivenko equivalent.

$(3) \Rightarrow(1)$. Since $\mathbf{K}$ and $\mathbf{L}$ are Glivenko equivalent, we have $\Phi \vdash_{\mathbf{K}} \sim-\psi$ iff $\Phi \vdash_{\mathbf{L}} \sim-\psi$. Moreover, since $\psi$ and $\sim-\psi$ are mutually deducible in $\mathbf{L}$, i.e. $\psi \vdash_{\mathbf{L}} \sim-\psi$ and $\sim-\psi \vdash_{\mathbf{L}} \psi$, we have that $\Phi \vdash_{\mathbf{L}} \sim-\psi$ is equivalent to $\Phi \vdash_{\mathbf{L}} \psi$.

Obviously, (4) implies (3). For the converse, if $\mathbf{L}$ is weakly involutive, then it is Glivenko involutive, so $\mathbf{L}=\mathbf{M}(\mathbf{K})$, by Proposition 4.1.

It is easy to see that the opposite of Proposition 4.4 is valid, so we obtain a characterization for the deductive Glivenko property, as well.

4.3. The equational Glivenko property. In view of Theorem 2.5 , it is clear that $\mathbf{K}$ has the left Glivenko property relative to $\mathbf{L}$ iff, for every term $t$

$$
\mathcal{V}(\mathbf{L}) \models 1 \leq t \text { iff } \mathcal{V}(\mathbf{K}) \models 1 \leq-\sim t .
$$

It is natural to consider the stronger property given by condition 5 of the following proposition.

Lemma 4.5. Let $\mathcal{W}, \mathcal{V}$ be subvarieties of $\mathcal{F} \mathcal{L}$ and let $s, t$ be terms over $\mathcal{F} \mathcal{L}$. Then, the following statements are equivalent. 
(1) $\mathcal{V}=s \approx t$ iff $\mathcal{W}=-\sim s \approx-\sim t$, for all $s, t$.

(2) $\mathcal{V}=s \approx t$ iff $\mathcal{W}=\sim s \approx \sim t$, for all $s, t$.

(3) $\mathcal{V}=s \leq t$ iff $\mathcal{W}=-\sim s \leq-\sim t$, for all $s, t$.

(4) $\mathcal{V}=s \leq t$ iff $\mathcal{W}=\sim s \geq \sim t$, for all $s, t$.

(5) $\mathcal{V} \mid=s \leq t$ iff $\mathcal{W}=s \leq-\sim t$, for all $s, t$.

The opposite statements are pairwise equivalent, as well.

Proof. The equivalences of (1) to (2) and of (3) to (4) follow from the fact that their right hand sides are equivalent, by Lemma 2.2. The same holds for the equivalence of (3) and (5), since by Lemma $2.2,-\sim s \leq-\sim t$ iff $s \leq-\sim t$. Moreover, it is clear that (3) implies (1). To show the converse it is enough to show that (2) implies (3). We assume that (2) holds. The inequality $s \leq t$ is valid in $\mathcal{V}$ iff the equation $s \vee t \approx t$ is valid in $\mathcal{V}$. By (2) this is the case exactly when $\mathcal{W}$ satisfies the equation $\sim(s \vee t) \approx \sim t$; i.e, by Lemma 2.2(1), when $\mathcal{W}$ satisfies $\sim s \wedge \sim t \approx \sim t$. The last equation is in turn equivalent to $\sim t \leq \sim s$, which, by Lemma 2.2(2) and (4), is equivalent to $-\sim s \leq-\sim t$.

To show that (1) implies (3), assume that (1) holds. The inequality $s \leq t$ is valid in $\mathcal{V}$ iff the equation $s \vee t \approx t$ is valid in $\mathcal{V}$. By (1) this is the case exactly when $\mathcal{W}$ satisfies $-\sim(s \vee t) \approx-\sim t$. By Lemma $2.2(5)$, the last equation is equivalent to $\sim(s \vee t) \approx \sim t$ and, by Lemma 2.2(1), it is equivalent to $\sim s \wedge \sim t \approx \sim t$. The last equation is in turn equivalent to $\sim t \leq \sim s$, which, by Lemma 2.2(2) and (4), is equivalent to $-\sim s \leq-\sim t$.

Let $\mathcal{W}$ and $\mathcal{V}$ be subvarieties of $\mathcal{F} \mathcal{L}$. We say that the left (right) equational Glivenko property holds for $\mathcal{W}$ relative to $\mathcal{V}$, if any of the statements (1)(5) (the opposite statements of (1)-(5), respectively) of the previous lemma holds. If both the left and the right equational Glivenko property hold for $\mathcal{W}$ relative to $\mathcal{V}$, we say that the equational Glivenko property holds for $\mathcal{W}$ relative to $\mathcal{V}$. The definition for substructural logics refers to the corresponding varieties. The following results have obvious analogues for substructural logics.

Proposition 4.6. Let $\mathcal{W}$ and $\mathcal{V}$ be subvarieties of $\mathcal{F} \mathcal{L}$. Then, the following statements are equivalent.

(1) The left equational Glivenko property holds for $\mathcal{W}$ relative to $\mathcal{V}$.

(2) $\mathcal{W}$ and $\mathcal{V}$ are Glivenko equivalent and $\mathcal{V}$ is left involutive.

(3) $\mathcal{V}=\mathbf{M}(\mathcal{W})$ and $\mathbf{M}(\mathcal{W})$ is left involutive.

Proof. Assume that the left equational Glivenko property holds for $\mathcal{W}$ relative to $\mathcal{V}$. By Lemma $2.2, \mathcal{W}$ satisfies $-\sim-\sim x \approx-\sim x$, so $\mathcal{V}$ satisfies $-\sim x \approx x$; i.e., $\mathcal{V}$ is left involutive. Moreover, the variety $\mathcal{V}$ satisfies $-s \approx-t$ iff $\mathcal{W}$ satisfies $-\sim-s \approx-\sim-t$, by (1) of Lemma 4.5, iff $\mathcal{W}$ satisfies $-s \approx-t$, by Lemma 2.2(4). Thus, (2) of Lemma 3.1 holds; i.e., $\mathcal{V}$ and $\mathcal{W}$ are Glivenko equivalent.

Conversely, assume that $\mathcal{V}$ is left involutive and that statement (2) of Lemma 3.1 of holds; i.e. assume that 


$$
\mathcal{V} \models-s \approx-t \text { iff } \mathcal{W} \models-s \approx-t .
$$

We will show that (1) of Lemma 4.5 holds, as well. If $\mathcal{V}$ satisfies $s \approx t$, then it also satisfies $-\sim s \approx-\sim t$. Thus, $\mathcal{W}$ satisfies $-\sim s \approx-\sim t$, by (2) of Lemma 3.1. Conversely, if $\mathcal{W}$ satisfies $-\sim s \approx-\sim t$, then $\mathcal{V}$ satisfies $-\sim s \approx-\sim t$, by (2) Lemma 3.1. So, $\mathcal{V}$ satisfies $s \approx t$, since $\mathcal{V}$ is left involutive.

Obviously, (3) implies (2). Conversely, if $\mathcal{V}$ is left involutive, then it is left Glivenko involutive, so by the algebraic analogue of Proposition 4.1, we have $\mathcal{V}=\mathbf{M}(\mathcal{W})$

We summarize the previous results in the following corollary.

Corollary 4.7. Let $\mathcal{W}, \mathcal{V}$ and $\mathcal{U}$ be subvarieties of $\mathcal{F} \mathcal{L}$ and consider the three properties for $\mathcal{W}$ relative to $\mathcal{V}$ - the (left) Glivenko property, deductive Glivenko property and equational Glivenko property - and the corresponding notions of involutiveness for $\mathcal{V}$ - (left) Glivenko involutive, weakly involutive and involutive.

(1) A Glivenko property holds for $\mathcal{W}$ relative to $\mathcal{V}$ iff $\mathcal{V}$ possesses the corresponding type of involutiveness and $\mathcal{V} \subseteq \mathcal{W} \subseteq \mathbf{G}(\mathcal{V})$.

(2) If $\mathcal{V}$ and $\mathcal{W}$ are Glivenko equivalent, then the left version of a Glivenko property holds for $\mathcal{V}$ relative to $\mathcal{U}$ iff it holds for $\mathcal{W}$ relative to $\mathcal{U}$.

(3) If $\mathcal{V}$ possesses the left version of a type of involutiveness, then the corresponding right Glivenko property for $\mathcal{W}$ relative to $\mathcal{V}$ implies the corresponding left Glivenko property for $\mathcal{W}$ relative to $\mathcal{V}$.

(4) In particular, if $\mathcal{V}$ possesses both the left and right versions of a type of involutiveness, then the left and right versions of the corresponding Glivenko property for $\mathcal{W}$ relative to $\mathcal{V}$ are mutually equivalent.

Proof. All statements are clear, if one recalls that, by Proposition 4.1 and the algebraization result, if $\mathcal{V}$ is even (left) Glivenko involutive, then $\mathbf{M}(\mathcal{V})=$ $\mathcal{V}$.

Corollary 4.8. If $\mathcal{V}$ is a left involutive or right involutive subvariety of $\mathcal{F} \mathcal{L}$ and there exists a variety $\mathcal{W}$ with a decidable equational theory, such that $\mathcal{V} \subseteq \mathcal{W} \subseteq \mathbf{G}(\mathcal{V})$, then $\mathcal{V}$ has a decidable equational theory, as well.

We have shown that the equational Glivenko property implies the deductive Glivenko property; also, the later implies the Glivenko property. We will provide examples that show that the converse of these implications do not hold. We say that a property holds for a subvariety of $\mathcal{F} \mathcal{L}$ or a substructural logic, if the property holds for the variety or the logic with respect to some variety or logic.

It follows from Proposition 4.1, Proposition 4.4 and Proposition 4.6 that a variety or logic has a certain type of involutiveness iff it satisfies the corresponding Glivenko property with respect to itself. We will make use of this remark in the proofs of the following two propositions. 
Proposition 4.9. The variety $\mathcal{C} \mathcal{F} \mathcal{L}$ (the logic $\mathbf{F L}_{e}$ ) is Glivenko involutive, but not weakly involutive. In other words, the Glivenko property holds for $\mathcal{C F} \mathcal{L}\left(\mathbf{F L}_{e}\right)$, but the deductive Glivenko property fails.

Proof. Note that $\sim \sim \phi$ is provable in $\mathbf{F L}_{e}$ iff $\phi$ is provable in $\mathbf{F L} \mathbf{L}_{e}$. This follows from the cut elimination theorem for $\mathbf{F L}_{e}$. In detail, if $\Rightarrow(\phi \rightarrow$ $0) \rightarrow 0$ is the last sequent in a proof in $\mathbf{F} \mathbf{L}_{e}$, then the only possibility for the upper sequent of the last rule is $\phi \rightarrow 0 \Rightarrow 0$. In turn, the only possibilities for the upper sequents of the next to the last rule are $\Rightarrow \phi$ and $0 \Rightarrow 0$. Consequently, $\mathcal{C F} \mathcal{L}$ is Glivenko involutive. Therefore, the Glivenko property holds for $\mathcal{C} \mathcal{F} \mathcal{L}\left(\mathbf{F L}_{e}\right)$, relative to itself, by Proposition 4.1.

On the other hand, if $\mathbf{F L}_{e}$ satisfies the deductive Glivenko property, then $\sim \sim p \vdash_{\mathbf{F L}_{e}} p$, where $p$ is a propositional variable. By the local deduction theorem, we have that for some $n, \vdash_{\mathbf{F L}}(\sim \sim p \wedge 1)^{n} \rightarrow p$. Nevertheless, there is a commutative pointed residuated lattice that does not satisfy the identity $(\sim \sim x \wedge 1)^{n} \leq x$, for any $n$. Indeed, consider the two-element residuated lattice on the set $\{\perp, 1\}$, where $\perp<1$, multiplication is idempotent and commutative, and 1 is the unit element. If we chose $0=1$, then we have $(\sim \sim \perp \wedge 1)^{n}=(1 \wedge 1)^{n}=1$, for all $n$.

Proposition 4.10. The variety $\mathcal{C} \mathcal{F} \mathcal{L} \cap \operatorname{Mod}\left((\sim \sim x)^{2} \leq x\right)$ (the logic $\mathbf{F L}_{e}+$ $\left.(\sim \sim p)^{2} \rightarrow p\right)$ is weakly involutive, but not involutive. In other words, The deductive Glivenko property holds for it, but the equational Glivenko property fails.

Proof. It follows from the local deduction theorem that the variety $\mathcal{V}=$ $\mathcal{C F} \mathcal{L} \cap \operatorname{Mod}\left((\sim \sim x)^{2} \leq x\right)$ is weekly involutive. Consequently, $\mathcal{V}=\mathbf{M}(\mathcal{V})$ and the deductive Glivenko property holds for $\mathcal{V}$ relative to itself, by Proposition 4.4 .

The equational Glivenko property holds for $\mathcal{V}=\mathbf{M}(\mathcal{V})$ iff it is involutive, by Proposition 4.6. This is not the case, since there exists a commutative pointed residuated lattice that satisfies the identity $(\sim \sim x)^{2} \leq x$, but is not involutive. Indeed, consider the residuated lattice on the set $\{\perp, a, 1\}$, where $\perp<a<1,1$ is the unit, $\perp$ is an absorbing element and $a^{2}=\perp$. If we chose $0=a$, then $\sim \sim \perp=a$, so the algebra is not involutive. Nevertheless, $(\sim \sim x)^{2}=x$, for every $x \in\{\perp, a, 1\}$.

4.4. The Glivenko variety of an involutive variety. Next, given an equational basis of an involutive variety $\mathcal{V}$, we show how to obtain an explicit axiomatization of the Glivenko variety $\mathbf{G}(\mathcal{V})$ of $\mathcal{V}$. Recall that $\mathbf{G}(\mathcal{V})$ was defined on page 11. Equivalently, given an axiomatization of an involutive substructural logic $\mathbf{L}$, we give an explicit axiomatization of the Glivenko $\operatorname{logic} \mathbf{G}(\mathbf{L})$ of $\mathbf{L}$.

The subvariety $\mathcal{G} l$ of $\mathcal{F} \mathcal{L}$ axiomatized by the equations

$$
\sim(x \star y) \approx \sim(-\sim x \star-\sim y)
$$


where $\star \in\{\wedge, \cdot, \backslash, /\}$, is called the left Glivenko variety. Also, the subvariety $\mathcal{G} r$ of $\mathcal{F} \mathcal{L}$ axiomatized by the equations

$$
-(x \star y) \approx-(\sim-x \star \sim-y),
$$

where $\star \in\{\wedge, \cdot, \backslash, /\}$, is called the right Glivenko variety. The variety $\mathcal{G}=$ $\mathcal{G} l \cap \mathcal{G} r$ is called the Glivenko variety. We will show, see Proposition 4.11(3), that the (left-, right-) Glivenko variety is the (left-, right-) Glivenko variety of the largest (left-, right-) involutive subvariety of $\mathcal{F} \mathcal{L}$; see page 24 for the definition. Note that, by Lemma 2.1(3) and Lemma 2.2(4), the equations $(\mathrm{Gl})$ and $(\mathrm{Gr})$ for $\star=\vee$ hold in all subvarieties of $\mathcal{F} \mathcal{L}$, thus we do not include them in the axiomatization of the left Glivenko and right Glivenko variety.

For every subvariety $\mathcal{V}$ of $\mathcal{F} \mathcal{L}$, and for every equational basis $B=\left\{s_{i} \approx\right.$ $\left.t_{i} \mid i \in I\right\}$ of $\mathcal{V}$ relative to $\mathcal{F} \mathcal{L}$, let $\mathcal{V}^{B}\left(\mathcal{V}_{B}\right.$, respectively) be the subvariety of $\mathcal{G} l\left(\mathcal{G} r\right.$, respectively) axiomatized by the equations $\sim s_{i} \approx \sim t_{i}\left(-s_{i} \approx-t_{i}\right.$, respectively), where $i \in I$, We will show that if $\mathcal{V}$ is a subvariety of $\mathcal{G}$, then $\mathcal{V}_{B}$ and $\mathcal{V}^{B}$ are equal to $\mathbf{G}(\mathcal{V})$. Thus, we obtain an explicit axiomatization of $\mathbf{G}(\mathcal{V})$ relative to $\mathcal{F} \mathcal{L}$.

For every pointed residuated lattice $\mathbf{A}$, define the binary relations $\lambda, \rho$ and $\theta$, by $x \lambda y$ iff $\sim x=\sim y, x \rho y$ iff $-x=-y$, and $x \theta y$ iff both $x \lambda y$ and $x \rho y$, for all $x, y \in A$. Obviously, $\lambda, \rho$ and $\theta$ are equivalence relations on $A$.

Proposition 4.11. Assume that $\mathcal{V}$ is a subvariety of $\mathcal{F} \mathcal{L}, B$ an equational basis of $\mathcal{V}$ and $\mathbf{A}$ a pointed residuated lattice.

(1) The implications (a) $\Rightarrow$ (b) $\Rightarrow$ (c) hold for the following statements.

(a) $\mathbf{A}$ is in $\mathcal{V}^{B}\left(\mathcal{V}_{B}, \mathcal{V}^{B} \cap \mathcal{V}_{B}\right.$, respectively).

(b) $\lambda(\rho, \theta$, respectively) is a congruence relation on $\mathbf{A}$ and $\mathbf{A} / \lambda$ $(\mathbf{A} / \rho, \mathbf{A} / \theta$, respectively) is in $\mathcal{V}$,

(c) $\mathbf{A}$ is in $\mathbf{G}(\mathcal{V})$.

Consequently, $\mathcal{V}_{B} \subseteq \mathbf{G}(\mathcal{V})$ and $\mathcal{V}^{B} \subseteq \mathbf{G}(\mathcal{V})$

(2) If $\mathbf{A}$ is in $\mathbf{G}(\mathcal{V})$ and $\lambda(\rho, \theta$, respectively) is a congruence relation on $\mathbf{A}$, then $\mathbf{A} / \lambda(\mathbf{A} / \rho, \mathbf{A} / \theta$, respectively) is in $\mathcal{V}$.

(3) If $\mathcal{V}$ is a subvariety of $\mathcal{G l}(\mathcal{G} r, \mathcal{G})$, then the corresponding statements in (a)-(c) of (1) are equivalent. In particular, $\mathcal{V}^{B}=\mathbf{G}(\mathcal{V})\left(\mathcal{V}_{B}=\right.$ $\mathbf{G}(\mathcal{V}), \mathcal{V}_{B}=\mathcal{V}^{B}=\mathbf{G}(\mathcal{V})$, respectively).

(4) If $\mathcal{V}$ is a finitely axiomatized subvariety of $\mathcal{G l}$ or of $\mathcal{G} r$, then so is $\mathbf{G}(\mathcal{V})$.

Proof. For the first implication in (1), assume that $\mathbf{A}$ is in $\mathcal{V}^{B}$. By the definitions of the relation $\lambda$ and the variety $\mathcal{V}^{B}$ and by (1) and (4) of Lemma 2.2, it is clear that $\lambda$ is a congruence on $\mathbf{A}$. Consequently, $\mathbf{A} / \lambda$ is a residuated lattice. Note that $\mathbf{A} / \lambda$ satisfies all the equations in $B$, by the definition of the variety $\mathcal{V}^{B}$; hence $\mathbf{A} / \lambda \in \mathcal{V}$. 
For the second implication, assume that $\lambda$ is a congruence relation on $\mathbf{A}$ and $\mathbf{A} / \lambda$ is in $\mathcal{V}$. If the equation $s \approx t$ holds in $\mathcal{V}$, then it also holds in $\mathbf{A} / \lambda$; hence, the equation $\sim s \approx \sim t$ is valid in $\mathbf{A}$. Consequently, $\mathbf{A}$ is in $\mathbf{G}(\mathcal{V})$.

For $(2)$, let $\mathbf{A}$ be in $\mathbf{G}(\mathcal{V})$ and let $\lambda$ be a congruence on $\mathbf{A}$. If $s \approx t$ holds in $\mathcal{V}$, then $\sim s \approx \sim t$ holds in $\mathbf{G}(\mathcal{V})$, hence also in $\mathbf{A}$. So, $s \approx t$ holds in $\mathbf{A} / \lambda$. Thus, $\mathbf{A} / \lambda \in \mathcal{V}$.

In view of (1), it suffices to show the implication $(c) \Rightarrow$ (a) in order to establish (3). If $\mathcal{V}$ is a subvariety of $\mathcal{G l}$, then it satisfies the equations (Gl). Since, $\mathbf{G}(\mathcal{V})$ is Glivenko equivalent to $\mathcal{V}$, it satisfies the equations (Gl), as well. Consequently, $\mathbf{G}(\mathcal{V})$ satisfies all the equations in the axiomatization of $\mathcal{V}^{B}$; thus, $\mathbf{G}(\mathcal{V}) \subseteq \mathcal{V}^{B}$. Finally, statement (4) follows from (3).

We define the left Glivenko logic $\mathbf{G l}=\mathbf{L}(\mathcal{G l})$, the right Glivenko logic $\mathbf{G r}=\mathbf{L}(\mathcal{G r})$ and the Glivenko logic $\mathbf{G l}=\mathbf{L}(\mathcal{G})$. We restate the main result in Proposition 4.11 in the terminology of logic.

Corollary 4.12. If a logic $\mathbf{L}$ is an extension of $\mathbf{G l}$ axiomatized by a set of formulas $\Phi$, then $\mathbf{G}(\mathbf{L})$ is axiomatized by

$$
\{\sim-\phi \mid \phi \in \Phi\} \cup\{(\sim(\phi \star \psi)) /(\sim(-\sim \phi \star-\sim \psi)) \mid \star \in\{\wedge, \cdot, \backslash, /\}\},
$$

or by the opposite formulas.

Proof. Note that $\mathcal{V}(\mathbf{L})$ is a subvariety of $\mathcal{G l}$ axiomatized by $\{\phi \approx \phi \vee 1 \mid \phi \in$ $\Phi\}$. By Proposition 4.11, $\mathbf{G}(\mathcal{V}(\mathbf{L}))$ is axiomatized by $\{\sim \phi \approx \sim(\phi \vee 1) \mid \phi \in$ $\Phi\} \cup(\mathrm{Gl})$, or equivalently by $\{1 \leq-\sim \phi \mid \phi \in \Phi\} \cup(\mathrm{Gl})$.

Corollary 4.13. If a logic $\mathbf{L}$ is a finitely axiomatized extension of $\mathbf{G l}$, then $\mathbf{G}(\mathbf{L})$ is also finitely axiomatized.

For example, if $\mathbf{L}$ is left involutive and finitely axiomatized, we can give an explicit axiomatization of the smallest logic for which the Glivenko property holds relative to $\mathbf{L}$. We will give some interesting such examples in Section 6 .

\section{More on the equational Glivenko property}

5.1. The deductive equational Glivenko property. In this section, we show that the deductive form of the equational Glivenko property is equivalent to the equational Glivenko property.

Lemma 5.1. Let $\mathcal{W}$ and $\mathcal{V}$ be subvarieties of $\mathcal{F} \mathcal{L}$ and let $E \cup\{s \approx t\}$ be a set of equations in the language of $\mathcal{F} \mathcal{L}$. Then, the following statements are equivalent.

(1) $E \models \mathcal{V} s \approx t$ iff $E \models \mathcal{W}-\sim s \approx-\sim t$.

(2) $E \models \mathcal{V} s \approx t$ iff $E \models \mathcal{W} \sim s \approx \sim t$.

(3) $E \models \mathcal{V} s \leq t$ iff $E \models \mathcal{W}-\sim s \leq-\sim t$.

(4) $E \models \mathcal{V} s \leq t$ iff $E \models \mathcal{W} \sim s \geq \sim t$.

(5) $E \models \mathcal{V} s \leq t$ iff $E \models \mathcal{W} s \leq-\sim t$.

The opposite statements are pairwise equivalent, as well. 
Proof. The proof is similar to the proof of Lemma 4.5, which is a special case for $E=\emptyset$, and is left to the reader.

Let $\mathcal{W}$ and $\mathcal{V}$ be subvarieties of $\mathcal{F} \mathcal{L}$. We say that the left (right) deductive equational Glivenko property holds for $\mathcal{W}$ relative to $\mathcal{V}$, if, for every set $E \cup\{s \approx t\}$ of equations in the language of $\mathcal{F} \mathcal{L}$, the statements (the opposite of the statements, respectively) in the first set of Lemma 5.1 hold. We say that the deductive equational Glivenko property holds for $\mathcal{W}$ relative to $\mathcal{V}$, if both left and right deductive equational Glivenko properties hold.

A map $\gamma$ on a (pointed) residuated lattice $\mathbf{A}$ is called a nucleus, if it is a closure operator on $\mathbf{A}$ and, for all $x, y \in A, \gamma(x) \gamma(y) \leq \gamma(x y)$. For equivalent definitions, see [12].

Consider the pointed residuated lattice terms $\lambda(x)=-\sim x$ and $\rho(x)=$ $\sim-x$. We will use the same symbols for the term operations that these terms define on particular pointed residuated lattices. Recall the binary relation $\lambda$ defined in the previous section and note that, if $\mathbf{A}$ is a pointed residuated lattice and $x, y \in A$, then $x \lambda y$ iff $\lambda(x)=\lambda(y)$. In other words, we use the same symbol for the map and its kernel.

Lemma 5.2. If $\mathbf{A}$ is a pointed residuated lattice, then the maps $\lambda$ and $\rho$ are closure operators on $\mathbf{A}$. If for all $x \in \mathbf{A},(x \backslash 0) \backslash 0=0 /(x \backslash 0) \quad((x \backslash 0) \backslash 0=$ $0 /(x \backslash 0))$, then $\lambda(\rho$, respectively) is a nucleus on $\mathbf{A}$. In particular, if $\mathbf{A}$ is cyclic, then $\lambda$ and $\rho$ are nuclei on $\mathbf{A}$.

Proof. The fact that $\lambda$ is a closure operator follows from (2), (3) and (4) of Lemma 2.2. Using Lemma 2.1(4) and (13), we have

$$
\begin{aligned}
{[y \backslash(x \backslash 0)] \lambda(x) \lambda(y) } & =[y \backslash(x \backslash 0)][0 /(x \backslash 0)][0 /(y \backslash 0)] \\
& =[y \backslash(x \backslash 0)][(x \backslash 0) \backslash 0][(y \backslash 0) \backslash 0] \\
& \leq[y \backslash 0][(y \backslash 0) \backslash 0] \leq 0 .
\end{aligned}
$$

So, we have $\lambda(x) \lambda(y) \leq[y \backslash(x \backslash 0)] \backslash 0=(x y \backslash 0) \backslash 0=0 /(x y \backslash 0)=\lambda(x y)$, by Lemma 2.1(6) and the assumption.

Lemma 5.3. If $\gamma$ is a nucleus on a pointed residuated lattice $\mathbf{A}$, then the algebra $\mathbf{A}_{\gamma}=\left\langle\gamma(A), \wedge, \vee_{\gamma},{ }_{\gamma}, \backslash, /, \gamma(1), \gamma(0)\right\rangle$, where $x \cdot{ }_{\gamma} y=\gamma(x y)$ and $x \vee_{\gamma}$ $y=\gamma(x \vee y)$, is a pointed residuated lattice. If, additionally, $\gamma$ is either $\lambda$ or $\rho$, then $\gamma(0)=0$ and $\mathbf{A}_{\gamma}$ is left involutive or right involutive, respectively.

Proof. It is shown in [12], see also [10], and in [16] for the commutative case, that the algebra $\left\langle\gamma(A), \wedge, \vee_{\gamma},{ }^{\prime} \gamma, \backslash, /, \gamma(1)\right\rangle$ is a residuated lattice. Thus, $\mathbf{A}_{\gamma}$ is a pointed residuated lattice. Moreover, $\lambda(0)=0$, by Lemma 2.1(12).

Theorem 5.4. Let $\mathcal{V}$ and $\mathcal{W}$ be subvarieties of $\mathcal{F} \mathcal{L}$. Then, the following statements are equivalent.

(1) The left equational Glivenko property holds for $\mathcal{W}$ relative to $\mathcal{V}$.

(2) The left deductive equational Glivenko property holds for $\mathcal{W}$ relative to $\mathcal{V}$. 
(3) $E \models \mathcal{V} s \approx t$ iff $-\sim E \models \mathcal{W}-\sim s \approx-\sim t$, for all $E, s, t$.

Proof. By taking $E$ to be the empty set in (3), we obtain the left equational Glivenko property; so (3) implies (1).

We will show that (2) implies (3). Suppose that (2) holds and assume that $E \models \mathcal{V} s \approx t$. Then, $-\sim E \mid=\mathcal{V} s \approx t$, since $\mathcal{V}$ is left involutive by Proposition 4.6. By (2), we have $-\sim E \models \mathcal{W}-\sim s \approx-\sim t$. Conversely, assume that $-\sim E \mid \mathcal{W}-\sim s \approx-\sim t$. Since $E \mid=\mathcal{W}-\sim u \approx-\sim v$, for all $(u \approx v) \in E$, we have $E \models_{\mathcal{W}}-\sim s \approx-\sim t$. By $(2)$, we get $E \models_{\mathcal{W}} s \approx t$. Thus, (2) implies (3). We will prove that (1) implies (2).

We will first show that, for all $\mathbf{A} \in \mathcal{W}, \lambda$ is a homomorphism from $\mathbf{A}$ onto $\mathbf{A}_{\lambda}$. By Proposition 4.6, Proposition 4.11(3) and Lemma 2.2, $\mathbf{G}(\mathcal{V})$ satisfies the equations $\sim(x \star y) \approx \sim(-\sim x \star-\sim y)$, hence also the equations $-\sim(x \star y) \approx-\sim(-\sim x \star-\sim y)$ where $\star \in\{\wedge, \cdot, \backslash, /\}$. Consequently, the latter set of equations holds in $\mathbf{A}$, since $\mathcal{W} \subseteq \mathbf{G}(\mathcal{V})$; the last inclusion follows from Lemma 3.3(4) and the fact that, by Proposition 4.6, the varieties $\mathcal{V}$ and $\mathcal{W}$ are Glivenko equivalent. For $\star=$, we have $\lambda(x) \cdot \lambda(y) \leq \lambda(\lambda(x) \cdot \lambda(y))=$ $\lambda(x y)$, for all $x, y \in A$; so, in view of Lemma $5.2, \lambda$ is a nucleus from $\mathbf{A}$ to $\mathbf{A}_{\lambda}$. Thus,

$$
\begin{gathered}
\lambda(x y)=\lambda(x) \cdot \mathbf{A}_{\lambda} \lambda(y), \lambda(1)=1_{\mathbf{A}_{\lambda}, \lambda(0)=0_{\mathbf{A}_{\lambda}} \text { and }} \\
\lambda(x \vee y)=\lambda(x) \vee_{\mathbf{A}_{\lambda}} \lambda(y),
\end{gathered}
$$

for all $x, y \in A$, by Lemma 5.3. By the same lemma, $\mathbf{A}_{\lambda}$ is closed under the meet and division operations of $\mathbf{L}$. So, for $\star \in\{\wedge, \backslash, /\}$ we have

$$
\lambda(x \star y)=\lambda(\lambda(x) \star \lambda(y))=\lambda\left(\lambda(x) \star \mathbf{A}_{\lambda} \lambda(y)\right)=\lambda(x) \star_{\mathbf{A}_{\lambda}} \lambda(y) .
$$

Now, assume that $E \models \mathcal{V} s \approx t$, where $E=\left\{s_{i}(\bar{x}) \approx t_{i}(\bar{x}) \mid i \in I\right\}$. To show that $E \models_{\mathcal{W}}-\sim s \approx-\sim t$, let $\mathbf{A} \in \mathcal{W}$ and assume that, for all $i \in I$, $s_{i}^{\mathbf{A}}(\bar{a})=t_{i}^{\mathbf{A}}(\bar{a})$, where $\bar{a}$ is an element of the appropriate power of $\mathbf{A}$. We will show that $\lambda\left(s^{\mathbf{A}}(\bar{a})\right)=\lambda\left(t^{\mathbf{A}}(\bar{a})\right)$. Since $\lambda\left(s_{i}^{\mathbf{A}}(\bar{a})\right)=\lambda\left(t_{i}^{\mathbf{A}}(\bar{a})\right)$, for all $i \in I$, and since $\lambda$ is a homomorphism, we obtain $s_{i}^{\mathbf{A}_{\lambda}}(\lambda(\bar{a}))=t_{i}^{\mathbf{A}_{\lambda}}(\lambda(\bar{a}))$, where $\lambda(\bar{a})$ denotes the sequence consisting of the $\lambda$ images of the terms in $\bar{a}$. Note that $\mathbf{A}_{\lambda}$ is in $\mathcal{W}$, since it is a homomorphic image of an algebra in $\mathcal{W}$; hence $\mathbf{A}_{\lambda} \in \mathbf{G}(\mathcal{V})$, since $\mathcal{W} \subseteq \mathbf{G}(\mathcal{V})$. If $u \approx v$ is an equation valid in $\mathcal{V}$, then the equation $\sim u \approx \sim v$ holds in $\mathbf{G}(\mathcal{V})$ and hence it holds in $\mathbf{A}_{\lambda}$. By Lemma 5.3, $\mathbf{A}_{\lambda}$ is left involutive, so the equation $s \approx t$ holds in $\mathbf{A}_{\lambda}$. Consequently, $\mathbf{A}_{\lambda}$ is in $\mathcal{V}$, hence it satisfies $s^{\mathbf{A}_{\lambda}}(\lambda(\bar{a}))=t^{\mathbf{A}_{\lambda}}(\lambda(\bar{a}))$. Thus, $\lambda\left(s^{\mathbf{A}}(\bar{a})\right)=\lambda\left(t^{\mathbf{A}}(\bar{a})\right)$, since $\lambda$ is a homomorphism. Consequently, $E \models \mathcal{W}-\sim s \approx-\sim t$. Conversely, suppose that $E \models \mathcal{W}-\sim s \approx-\sim t$. By Corollary 4.7, $\mathcal{V} \subseteq \mathcal{W}$, so $E=\mathcal{V}-\sim s \approx-\sim t$. Since $\mathcal{V}$ is left involutive, we have $E \mid=\mathcal{V} s \approx t$.

Corollary 5.5. If $\mathcal{V}$ is an involutive subvariety of $\mathcal{F} \mathcal{L}$ and the quasi-equational theory of a variety $\mathcal{W}$, where $\mathcal{V} \subseteq \mathcal{W} \subseteq \mathbf{G}(\mathcal{V})$, is decidable, then the quasiequational theory of $\mathcal{V}$ is decidable, as well. 
5.2. An alternative characterization for the equational Glivenko property. We have obtained a characterization of the Glivenko properties in terms of the type of involutiveness that the minimal variety of the Glivenko equivalence class has to possess. Here we describe varieties, containment in which guarantees the validity of the equational Glivenko property.

Let $\mathcal{I} l \mathcal{F} \mathcal{L}$ (respectively, $\mathcal{I} r \mathcal{F} \mathcal{L}$ ) be the variety of left- (right-) involutive pointed residuated lattices, i.e. the subvariety of $\mathcal{F} \mathcal{L}$ axiomatized by the equation $-\sim x \approx x$ (respectively, $\sim-x \approx x$ ). Also, let $\mathcal{I} n \mathcal{F} \mathcal{L}=$ $\mathcal{I} l \mathcal{F} \mathcal{L} \cap \mathcal{I} r \mathcal{F} \mathcal{L}$. By Proposition 4.11(3), it follows that $\mathcal{G} l=\mathbf{G}(\mathcal{I} l \mathcal{F} \mathcal{L})$, $\mathcal{G} r=\mathbf{G}(\mathcal{I} r \mathcal{F} \mathcal{L})$ and $\mathcal{G}=\mathbf{G}(\mathcal{I} n \mathcal{F} \mathcal{L})$.

Note that, by Proposition 4.11, $\mathbf{A} \in \mathcal{G} l(\mathbf{A} \in \mathcal{G} r, \mathbf{A} \in \mathcal{G})$ iff $\lambda(\rho, \theta$, respectively) is a congruence on $\mathbf{A}$ and $\mathbf{A} / \lambda$ is left involutive $(\mathbf{A} / \rho$ is right involutive, $\mathbf{A} / \theta$ is involutive, respectively).

For every subvariety $\mathcal{V}$ of $\mathcal{F} \mathcal{L}$, set $\operatorname{Il}(\mathcal{V})=\operatorname{Il} \mathcal{F} \mathcal{L} \cap \mathcal{V}, \operatorname{Ir}(\mathcal{V})=\operatorname{Ir} \mathcal{F} \mathcal{L} \cap \mathcal{V}$ and $\operatorname{In}(\mathcal{V})=\operatorname{In} \mathcal{F} \mathcal{L} \cap \mathcal{V}$ - the largest left involutive, right involutive and involutive subvariety of $\mathcal{V}$, respectively. Note that $\mathbf{I l}, \mathbf{I r}$ and $\mathbf{I n}$ are interior operators on $\mathbf{S}(\mathcal{F} \mathcal{L})$. A notion related to $\operatorname{In}(\mathcal{V})$ is also discussed in [5].

Lemma 5.6. Let $\mathcal{V}$ and $\mathcal{W}$ be subvarieties of $\mathcal{F} \mathcal{L}$.

(1) If $\mathcal{V}$ and $\mathcal{W}$ are Glivenko equivalent, then $\operatorname{Il}(\mathcal{V})=\mathbf{I l}(\mathcal{W}), \operatorname{Ir}(\mathcal{V})=$ $\operatorname{Ir}(\mathcal{W})$, and $\operatorname{In}(\mathcal{V})=\operatorname{In}(\mathcal{W})$

(2) $\mathbf{I l}(\mathcal{V})=\mathbf{I l}(\mathbf{G}(\mathcal{V})), \operatorname{Ir}(\mathcal{V})=\mathbf{I r}(\mathbf{G}(\mathcal{V}))$, and $\mathbf{I n}(\mathcal{V})=\mathbf{I n}(\mathbf{G}(\mathcal{V}))$

(3) If $\mathcal{V}$ is left involutive (right involutive, involutive), then $\mathbf{I l}(\mathbf{G}(\mathcal{V}))=$ $\mathcal{V}(\operatorname{Ir}(\mathbf{G}(\mathcal{V}))=\mathcal{V}, \mathbf{I n}(\mathbf{G}(\mathcal{V}))=\mathcal{V}$, respectively $)$

(4) The varieties $\mathbf{I}(\mathcal{V}), \operatorname{Ir}(\mathcal{V})$ and $\operatorname{In}(\mathcal{V})$ are subvarieties of $\mathbf{M}(\mathcal{V})$.

(5) $\mathbf{G}(\mathbf{I l}(\mathcal{V}))=\mathcal{G} l \cap \mathbf{G}(\mathcal{V}), \mathbf{G}(\mathbf{I r}(\mathcal{V}))=\mathcal{G} r \cap \mathbf{G}(\mathcal{V})$, and $\mathbf{G}(\mathbf{I n}(\mathcal{V}))=$ $\mathcal{G} \cap \mathbf{G}(\mathcal{V})$.

Proof. For (1), assume that $\mathcal{V}$ and $\mathcal{W}$ are Glivenko equivalent. If $\mathcal{V}$ satisfies $s \approx t$, then it also satisfies $\sim s \approx \sim t$. So, $\mathcal{W}$ satisfies $\sim s \approx \sim t$, hence it satisfies $-\sim s \approx-\sim t$. Consequently, $\mathbf{I l}(\mathcal{W})$ satisfies $s \approx t$. Thus, $\mathbf{I l}(\mathcal{W}) \subseteq$ $\mathcal{V}$, so $\mathbf{I l}(\mathcal{W}) \subseteq \mathbf{I l}(\mathcal{V})$. Likewise, we show the other inclusion, so $\mathbf{I l}(\mathcal{W})=$ $\operatorname{Il}(\mathcal{I})$.

Statement (2) follows from (1) and Lemma 3.3(2). Statement (3) is a direct consequence of (2). For statement (4), note that since $\mathcal{V}$ and $\mathbf{M}(\mathcal{V})$ are Glivenko equivalent, using (1) we obtain $\mathbf{I l}(\mathcal{V})=\mathbf{I l}(\mathbf{M}(\mathcal{V})) \subseteq \mathbf{M}(\mathcal{V})$.

To show (5), note that if a pointed residuated lattice $\mathbf{A}$ is in $\mathcal{G l} \cap \mathbf{G}(\mathcal{V})=$ $\mathbf{G}(\mathcal{I} l \mathcal{F} \mathcal{L}) \cap \mathbf{G}(\mathcal{V})$, then, by taking $\mathcal{I} l \mathcal{F} \mathcal{L}$ for $\mathcal{V}$ in Proposition $4.11(3)$, we have that $\lambda$ is a congruence relation on $\mathbf{A}$ and $\mathbf{A} / \lambda$ is in $\mathcal{I} l \mathcal{F} \mathcal{L}$. So by applying Proposition $4.11(2)$ to $\mathcal{V}$, we get that $\mathbf{A} / \lambda$ is in $\mathcal{V}$. Therefore, $\mathbf{A} / \lambda$ is in $\mathcal{I} l \mathcal{F} \mathcal{L} \cap \mathcal{V}=\mathbf{I l}(\mathcal{V})$. By Proposition 4.11(1) we get $\mathbf{A}$ is in $\mathbf{G}(\mathbf{I l}(\mathcal{V}))$. 
Conversely, if $\mathbf{A}$ is in $\mathbf{G}(\mathbf{I l}(\mathcal{V}))$, then $\lambda$ is a congruence relation on $\mathbf{A}$ and $\mathbf{A} / \lambda$ is in $\mathbf{I l}(\mathcal{V})=\mathcal{I} l \mathcal{F} \mathcal{L} \cap \mathcal{V}$, by Proposition 4.11(3). By applying Proposition 4.11(1) to $\mathcal{V}$ and to $\mathcal{I} l \mathcal{F} \mathcal{L}$, we have $\mathbf{A} \in \mathbf{G}(\mathcal{V})$ and $\mathbf{A} \in \mathbf{G}(\mathcal{I} l \mathcal{F} \mathcal{L})$. So, $\mathbf{A} \in \mathbf{G}(\mathcal{V}) \cap \mathbf{G}(\mathcal{I} l \mathcal{F} \mathcal{L})=\mathbf{G}(\mathbf{I l}(\mathcal{V}))$.

The following theorem shows that the equational Glivenko property holds for a subvariety $\mathcal{V}$ of $\mathcal{F} \mathcal{L}$ iff $\mathcal{V}$ is contained in the Glivenko variety.

Theorem 5.7. The following are equivalent.

(1) $\mathcal{V}$ is a subvariety of $\mathcal{G l}(\mathcal{G} r, \mathcal{G})$.

(2) $\mathbf{G}(\mathcal{V})$ is a subvariety of $\mathcal{G l}(\mathcal{G} r, \mathcal{G}$, respectively).

(3) $\mathbf{M}(\mathcal{V})$ is a subvariety of $\mathcal{G l}(\mathcal{G} r, \mathcal{G}$, respectively).

(4) $\mathbf{M}(\mathcal{V})$ is equal to $\mathbf{I l}(\mathcal{V})(\operatorname{Ir}(\mathcal{V}), \operatorname{In}(\mathcal{V})$, respectively).

(5) The left equational Glivenko property holds for $\mathcal{V}$ relative to $\mathbf{I l}(\mathcal{V})$ $(\operatorname{Ir}(\mathcal{V}), \operatorname{In}(\mathcal{V})$, respectively).

(6) The left equational Glivenko property holds for $\mathcal{V}$ relative to some variety.

(7) $\mathbf{M}(\mathcal{V})$ is a subvariety of $\mathcal{I} l \mathcal{F} \mathcal{L}(\mathcal{I} r \mathcal{F} \mathcal{L}, \mathcal{I} \mathcal{F} \mathcal{L}$, respectively $)$.

Proof. We will establish the implications $(1) \Rightarrow(2) \Rightarrow(3) \Rightarrow(4) \Rightarrow(5) \Rightarrow$ $(6) \Rightarrow(1)$, and the equivalence $(7) \Leftrightarrow(4)$. The implications $(2) \Rightarrow(3)$ and $(5) \Rightarrow(6)$ are clear and the equivalence $(7) \Leftrightarrow(4)$ follows from Lemma 5.6(4) and the fact that $\mathbf{M}(\mathcal{V}) \subseteq \mathcal{V}$.

$(1) \Rightarrow(2):$ If $\mathcal{V} \subseteq \mathcal{G} l=\mathbf{G}(\mathcal{I} l \mathcal{F} \mathcal{L})$, then $\mathbf{G}(\mathcal{V}) \subseteq \mathbf{G}(\mathbf{G}(\mathcal{I} l \mathcal{F} \mathcal{L}))=\mathcal{G} l$

$(3) \Rightarrow(4)$ : Since $\mathbf{M}(\mathcal{V}) \subseteq \mathbf{G}(\mathcal{V})$, using Lemma 5.6(5) we obtain $\mathbf{M}(\mathcal{V}) \subseteq$ $\mathbf{G}(\mathbf{I l}(\mathcal{V}))$. Moreover, $\mathbf{I l}(\mathcal{V}) \subseteq \mathbf{M}(\mathcal{V})$ by Lemma 5.6(4), so the left equational Glivenko property holds for $\mathbf{M}(\mathcal{V})$ relative to $\mathbf{I l}(\mathcal{V})$ by Corollary 4.7(1). Consequently, by Proposition 4.6, $\mathbf{M}(\mathcal{V})$ and $\mathbf{I l}(\mathcal{V})$ are Glivenko equivalent. Since $\mathbf{M}(\mathcal{V})$ is Glivenko equivalent to $\mathcal{V}$, and $\mathcal{V}$ and $\mathbf{I l}(\mathcal{V})$ are Glivenko equivalent, we obtain $\mathbf{M}(\mathcal{V})=\mathbf{I l}(\mathcal{V})$ by Proposition 4.6.

$(4) \Rightarrow(5)$ : Since $\mathbf{I l}(\mathcal{V})=\mathbf{M}(\mathcal{V})$ and $\mathbf{I l}(\mathcal{V})$ is involutive, $(5)$ follows from Proposition 4.6.

$(6) \Rightarrow(1)$ : If the left equational Glivenko property holds for $\mathcal{V}$ relative to some variety $\mathcal{U}$, then $\mathcal{U} \subseteq \mathcal{V} \subseteq \mathbf{G}(\mathcal{U})$ and $\mathcal{U}$ is left involutive, by Lemma 4.7(1). So, $\mathcal{U}=\mathbf{I l}(\mathcal{U})$ and $\mathcal{V} \subseteq \mathbf{G}(\mathbf{I l}(\mathcal{U}))$. Since $\mathbf{I l}(\mathcal{U}) \subseteq \mathcal{I l} \mathcal{F} \mathcal{L}$, we have $\mathbf{G}(\mathbf{I l}(\mathcal{U})) \subseteq \mathbf{G}(\mathcal{I} l \mathcal{F} \mathcal{L})=\mathcal{G} l$, by Lemma 3.3(1b). Thus, $\mathcal{V} \subseteq \mathcal{G l}$.

The equivalence of statements (2) and (3) of the preceding theorem implies that the Glivenko equivalence class $[\mathbf{M}(\mathcal{V}), \mathbf{G}(\mathcal{V})]$ of $\mathcal{V}$ is either contained in the principal order ideal of $\mathbf{S}(\mathcal{F} \mathcal{L})$ generated by $\mathcal{G l}(\mathcal{G} r, \mathcal{G}$, respectively), or it is completely disjoint from it. In the first case and only then the least variety $\mathbf{M}(\mathcal{V})$ (equivalently some variety) of the interval is contained in $\mathcal{I} l \mathcal{F} \mathcal{L}(\mathcal{I} r \mathcal{F} \mathcal{L}, \mathcal{I} n \mathcal{F} \mathcal{L}$, respectively), or equivalently it is equal to $\operatorname{Il}(\mathcal{V})$ $(\operatorname{Ir}(\mathcal{V}), \operatorname{In}(\mathcal{V})$ respectively). In the second case, the equational Glivenko property fails to hold for every variety in the interval.

Corollary 5.8. $\mathbf{G}(\mathbf{I l}(\mathcal{V}))$ is the largest subvariety of $\mathbf{G}(\mathcal{V})$ for which the left equational Glivenko property holds relative to some variety. 
Proof. By Theorem 5.7, the left equational Glivenko property holds for a subvariety $\mathcal{W}$ of $\mathbf{G}(\mathcal{V})$ relative to some variety iff $\mathcal{W} \subseteq \mathbf{G}(\mathcal{V})$ and $\mathcal{W} \subseteq \mathcal{G} l$. By Lemma 5.6(5), this is equivalent to $\mathcal{W} \subseteq \mathbf{G}(\mathbf{I l}(\mathcal{V}))$.

\section{Special CASes}

In this section we discuss some special cases for which the Glivenko properties holds and describe how Theorems 1.1 and ?? follow from our results.

As we have seen the left Glivenko (right Glivenko, Glivenko) variety $\mathcal{G l}$ $(\mathcal{G} r, \mathcal{G}$, respectively) is axiomatized by the equations $\sim(x \star y) \approx \sim(-\sim x \star$ $-\sim y)(-(x \star y) \approx-(\sim-x \star \sim-y)$, the combination of both sets of equations, respectively), where $\star \in\{\wedge, \cdot, \backslash, /\}$. Given an axiomatization of a left involutive (right involutive, involutive) variety $\mathcal{V}$, an axiomatization of the Glivenko variety $\mathbf{G}(\mathcal{V})$ of $\mathcal{V}$ - the largest subvariety of $\mathcal{F} \mathcal{L}$ for which the left Glivenko (right Glivenko, Glivenko, respectively) property holds relative to $\mathcal{V}$ - consists of the axiomatization of $\mathcal{G l}(\mathcal{G} r, \mathcal{G}$, respectively) mentioned above plus the left (right, left and right, respectively) negations of the equations in the axiomatization of $\mathcal{V}$.

Moreover, for every subvariety $\mathcal{W}$ of $\mathcal{F} \mathcal{L}$, an axiomatization of the variety $\mathbf{G}_{\mathcal{W}}(\mathcal{V})=\mathbf{G}(\mathcal{V}) \cap \mathcal{W}$ - the largest subvariety of $\mathcal{W}$ for which the left Glivenko (right Glivenko, Glivenko) property holds relative to the left involutive (right involutive, involutive, respectively) variety $\mathcal{V}$ - is obtained by combining an axiomatization of $\mathbf{G}(\mathcal{V})$ with one of $\mathcal{W}$. Below we give a number of varieties $\mathcal{W}$ for which the axiomatization of $\mathbf{G}_{\mathcal{W}}(\mathcal{V})$ relative to $\mathcal{W}$ can be simplified. In particular, we obtain simpler axiomatizations for $\mathcal{G} l_{\mathcal{W}}=\mathcal{G l} \cap \mathcal{W}, \mathcal{G} r_{\mathcal{W}}=$ $\mathcal{G} r \cap \mathcal{W}$ and $\mathcal{G}_{\mathcal{W}}=\mathcal{G} \cap \mathcal{W}$ relative to $\mathcal{W}$. Moreover, we study the cases where $\mathcal{V}$ is the variety of Boolean algebras. In this way we show how known results on the Glivenko theorem can be derived from our result.

6.1. The cyclic case. Let $\mathcal{C} y \mathcal{F} \mathcal{L}=\operatorname{Mod}(\sim x \approx-x)$ be the cyclic subvariety of $\mathcal{F} \mathcal{L}$. Before we proceed, we point out that the equation for one of the division operations in (Gl) can be simplified.

Lemma 6.1. In every cyclic pointed residuated lattice the equations

$$
1 \leq \sim \sim(\sim \sim y \backslash y) \text { and } \sim(x \backslash y) \approx \sim(\sim \sim x \backslash \sim \sim y)
$$

are equivalent. The same holds for the equations

$$
1 \leq \sim \sim(y / \sim \sim y) \text { and } \sim(y / x) \approx \sim(\sim \sim y / \sim \sim x) .
$$

Proof. Assume that $\sim(\sim \sim x \backslash \sim \sim y) \approx \sim(x \backslash y)$ holds. Then,

$$
\sim \sim(\sim \sim x \backslash \sim \sim y) \approx \sim \sim(x \backslash y)
$$

holds as well. By setting $x=\sim \sim y$ and using (3), (2) and (4) of Lemma 2.2, we obtain

$$
1 \leq \sim \sim 1 \leq \sim \sim(\sim \sim y \backslash \sim \sim y) \approx \sim \sim(\sim \sim \sim \sim y \backslash \sim \sim y) \approx \sim \sim(\sim \sim y \backslash y) .
$$

For the converse, note that $(x \backslash \sim \sim y)(\sim \sim y \backslash y) \leq x \backslash y \leq \sim \sim(x \backslash y)$, by Lemma 2.1(4,5) and by Lemma 2.2(4); so $\sim \sim y \backslash y \leq(x \backslash \sim \sim y) \backslash \sim \sim(x \backslash y)$, 
hence

$$
\sim \sim(\sim \sim y \backslash y) \leq \sim \sim[(x \backslash \sim \sim y) \backslash \sim \sim(x \backslash y)] .
$$

Moreover, by Lemma 2.2(12),

$$
\sim \sim[(x \backslash \sim \sim y) \backslash \sim \sim(x \backslash y)] \approx(x \backslash \sim \sim y) \backslash \sim \sim(x \backslash y)
$$

and, by hypothesis, $1 \leq \sim \sim(\sim \sim y \backslash y)$, so we have $1 \leq(x \backslash \sim \sim y) \backslash \sim \sim(x \backslash y)$, that is $x \backslash \sim \sim y \leq \sim \sim(x \backslash y)$. Now, since $\sim \sim x \backslash \sim \sim y \leq x \backslash \sim \sim y$, we have $\sim \sim x \backslash \sim \sim y \leq \sim \sim(x \backslash y)$, hence $\sim(\sim \sim x \backslash \sim \sim y) \geq \sim(x \backslash y)$. The converse inequality follows from (11) of Lemma 2.2 .

Note that $\mathcal{G}_{\mathcal{C} y \mathcal{F} \mathcal{L}}=\mathcal{G} l_{\mathcal{C} y \mathcal{F} \mathcal{L}}=\mathcal{G}_{\mathcal{C} y \mathcal{F} \mathcal{L}}$

Corollary 6.2. The variety $\mathcal{G}_{\mathcal{C} y \mathcal{F} \mathcal{L}}$ is axiomatized relative to $\mathcal{C} y \mathcal{F} \mathcal{L}$ by the conjunction of the following equations

(1) $1 \leq \sim \sim(y / \sim \sim y)$,

(2) $1 \leq \sim \sim(\sim \sim y \backslash y)$,

(3) $\sim(x \wedge y) \approx \sim(\sim \sim x \wedge \sim \sim y)$.

Proof. Recall that $\mathcal{G}_{\mathcal{C} y \mathcal{F} \mathcal{L}}$ is axiomatized by the equations $(\mathrm{Gl})$ in Section 4.4; see page 19. We will show that the identity for multiplication is redundant. For every $x, y$ in an algebra in the variety $\mathcal{G}_{\mathcal{C} y \mathcal{F} \mathcal{L}}$, we have

$$
\begin{aligned}
\sim(x y) & =y \backslash \sim x & & \\
& =y \backslash-x & & (\sim x \approx-x) \\
& =-\sim y \backslash-\sim-x & & (\text { Lemma 2.2(4,10)) } \\
& =\sim \sim y \backslash \sim \sim \sim x & & (\sim x \approx-x) \\
& =\sim(\sim \sim x \cdot \sim \sim y) & & (\text { Lemma 2.2(8)) }
\end{aligned}
$$

By Lemma 6.1, the identities for the division operations follow from (1) and (2).

Recall that by $\mathcal{C} \mathcal{F} \mathcal{L}$ we denote the variety of commutative pointed residuated lattices.

Corollary 6.3. $\mathcal{G}_{\mathcal{C F} \mathcal{L}}$ is axiomatized relative to $\mathcal{C} \mathcal{F} \mathcal{L}$ by the equations $1 \leq$ $\sim \sim(\sim \sim y \rightarrow y)$ and $\sim(x \wedge y) \approx \sim(\sim \sim x \wedge \sim \sim y)$.

Let $\mathcal{I F} \mathcal{L}$ denote the variety of integral pointed residuated lattices and set $\mathcal{I C} y \mathcal{F} \mathcal{L}=\mathcal{C} y \mathcal{F} \mathcal{L} \cap \mathcal{I} \mathcal{F} \mathcal{L}$.

Corollary 6.4. The variety $\mathcal{G}_{\mathcal{I C} y \mathcal{F} \mathcal{L}}$ is axiomatized relative to $\mathcal{I C} y \mathcal{F} \mathcal{L}$ by the equations $1 \leq \sim \sim(y / \sim \sim y)$ and $1 \leq \sim \sim(\sim \sim y \backslash y)$.

Proof. We will show that the equation for the meet operation in Corollary 6.2 is redundant. Using integrality we have,

$$
(x / \sim \sim x)(\sim \sim x \wedge \sim \sim y)(\sim \sim y \backslash y) \leq(x / \sim \sim x) \sim \sim x \leq x .
$$

Similarly,

$$
(x / \sim \sim x)(\sim \sim x \wedge \sim \sim y)(\sim \sim y \backslash y) \leq y
$$


so

$$
(x / \sim \sim x)(\sim \sim x \wedge \sim \sim y)(\sim \sim y \backslash y) \leq x \wedge y \leq \sim \sim(x \wedge y) .
$$

By applying Lemma 2.2(13) twice, we obtain

$$
\sim \sim(x / \sim \sim x)(\sim \sim x \wedge \sim \sim y) \sim \sim(\sim \sim y \backslash y) \leq \sim \sim(x \wedge y) .
$$

Since $1 \leq \sim \sim(x / \sim \sim x)$ and $1 \leq \sim \sim(\sim \sim y \backslash y)$, we have

$$
\sim \sim x \wedge \sim \sim y \leq \sim \sim(x \wedge y)
$$

By (2) and (4) of Lemma 2.2, we obtain

$$
\sim(x \wedge y) \leq \sim(\sim \sim x \wedge \sim \sim y)
$$

The converse inequality follows from (3) and (2) of Lemma 2.2.

The following corollary can also be obtained from Theorem 5.1 of [6].

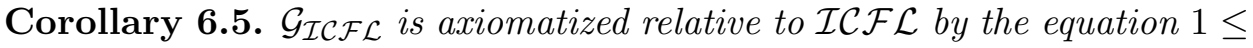
$\sim \sim(\sim \sim y \rightarrow y)$.

As a consequence we obtain Theorem ??(3).

In [6] Glivenko's Theorem 1.1 is generalized to logics containing BCKlogic. In algebraic terminology and in our notation it is shown that if $\mathcal{W}$ is a subquasivariety of a natural expansion of the quasivariety of bounded BCKalgebras that satisfies the equation $1 \leq \sim \sim(\sim \sim y \rightarrow y)$, then the Glivenko property holds for $\mathcal{W}$ relative to $\operatorname{In}(\mathcal{W})$. This result extends the original theorem to expansions of quasivarieties, but is limited to the integral, commutative case, where the negation constant 0 is the least element. Our result has exactly the opposite attributes and it extends to the stronger equational Glivenko property. Both results include the extension of Glivenko's theorem for commutative, integral, bounded pointed residuated lattices where 0 is the least element, given by Corollary 6.5.

6.2. The classical case. Note that a Brouwerian algebra is (term equivalent to) a residuated lattice that satisfies the law $x y \approx x \wedge y$. A Heyting algebra is (term equivalent to) a pointed residuated lattice, whose residuated lattice reduct is a Brouwerian algebra and 0 is its least element.

Lemma 6.6. A pointed residuated lattice is (term equivalent to) a Boolean algebra iff it satisfies the equations $x y \approx x \wedge y$ and $x \backslash y \approx \sim x \vee y$.

Proof. Setting $x=1$ into the second equation, we have $0 \leq y$. So, in view of the first equation the pointed residuated lattice is a Heyting algebra; hence it has a distributive lattice reduct. To show that it is a Boolean algebra, it suffices to show that every element has a complement. For every $x$, we have $x \wedge \sim x=x(x \backslash 0) \leq 0$, so $x \wedge \sim x=0$. Also, by the second equation $\sim x \vee x=x \backslash x=1$, since every Heyting algebra is integral.

In the case when the variety relative to which a Glivenko property holds is the variety $\mathcal{B A}$ of Boolean algebras, we obtain a simpler axiomatization. 
Corollary 6.7. $\mathbf{G}(\mathcal{B A})$ is axiomatized by the following equations.

(1) $\sim(x \wedge y) \approx \sim(x y)$

(2) $\sim(x \backslash y) \approx \sim(-x \vee y)$

(3) $-(x \backslash y) \approx-(\sim x \vee y)$

(4) $\sim(x \backslash y) \approx \sim(-\sim x \backslash-\sim y)$

(5) $\sim(x / y) \approx \sim(-\sim x /-\sim y)$

Proof. Given the axiomatization of $\mathcal{B A}$ in Lemma 6.6, an axiomatization of $\mathbf{G}(\mathcal{B A})$ consists of the equations (1), (2), (4) and (5) plus the equations $\sim(x \cdot y) \approx \sim(-\sim x \cdot-\sim y)$ and $\sim(x \wedge y) \approx \sim(-\sim x \wedge-\sim y)$. We will verify that these two equations follow from the proposed list. We have

$$
\begin{aligned}
\sim(-\sim x \wedge-\sim y) & =\sim-(\sim x \vee \sim y) \\
& =\sim-(x \backslash \sim y) \\
& =\sim-\sim(y x) \\
& =\sim(y x) \\
& =\sim(x \wedge y)
\end{aligned}
$$

Consequently, we have $\sim(x y)=\sim(x \wedge y)=\sim(-\sim x \wedge-\sim y)=\sim(-\sim x$. $-\sim y)$, as well.

Corollary 6.8. $\mathbf{G}_{\mathcal{C} y \mathcal{F} \mathcal{L}}(\mathcal{B A})$ is axiomatized relative to $\mathcal{C} y \mathcal{F} \mathcal{L}$ by the following equations.

(1) $\sim(x \wedge y) \approx \sim(x y)$

(2) $\sim(y / x) \approx \sim(\sim x \vee y)$

(3) $\sim(x \backslash y) \approx \sim(\sim x \vee y)$

Alternatively, (2) and (3) can be replaced respectively by

(4) $1 \leq \sim \sim(\sim \sim x \backslash x)$

(5) $1 \leq \sim \sim(x / \sim \sim x)$.

Proof. For the first axiomatization, in view of Corollary 6.7, it suffices to show that the equations for the division operations are redundant. We have

$$
\begin{aligned}
\sim(y / x) & =\sim(\sim x \vee y) \\
& =\sim \sim x \wedge \sim y \\
& =\sim \sim \sim \sim x \wedge \sim \sim \sim y \\
& =\sim(\sim \sim \sim x \vee \sim \sim y) \\
& =\sim(\sim \sim y / \sim \sim x)
\end{aligned}
$$

Likewise, $\sim(x \backslash y)=\sim(\sim \sim x \backslash \sim \sim y)$. Therefore, (1) and (2) form an axiomatization for $\mathbf{G}_{\mathcal{C} y \mathcal{F} \mathcal{L}}(\mathcal{B} \mathcal{A})$ relative to $\mathcal{C} y \mathcal{F} \mathcal{L}$.

Finally, we will show that (2) and (4) are equivalent; the equivalence of (3) and (5) follows in a similar way. If (2) holds, we have

$$
\begin{aligned}
\sim \sim(\sim \sim x \backslash x) & =\sim \sim(\sim \sim \sim x \vee x) \\
& =\sim \sim(\sim x \vee x) \\
& =\sim(\sim \sim x \wedge \sim x) \\
& =\sim(\sim \sim x \cdot \sim x) \\
& \geq \sim 0=1
\end{aligned}
$$


Conversely, assume that (4) holds. We have, $(x \backslash \sim \sim y)(\sim \sim y \backslash y) \leq x \backslash y$, by Lemma 2.1(13) and $x \backslash y \leq \sim \sim(x \backslash y)$, by Lemma 2.2(3), so

$$
(x \backslash \sim \sim y) \cdot(\sim \sim y \backslash y) \cdot \sim(x \backslash y) \leq 0 .
$$

By Lemma $2.3(3)$, we obtain $\sim \sim(x \backslash \sim \sim y) \cdot \sim \sim(\sim \sim y \backslash y) \cdot \sim \sim \sim(x \backslash y) \leq 0$, i.e. $\sim \sim(x \backslash \sim \sim y) \cdot \sim \sim(\sim \sim y \backslash y) \leq \sim \sim(x \backslash y)$. Using (4) and the fact that $\sim \sim x \backslash \sim \sim y \leq x \backslash \sim \sim y$, we obtain

$$
\sim \sim(\sim \sim x \backslash \sim \sim y) \leq \sim \sim(x \backslash y),
$$

thus $\sim(x \backslash y) \leq \sim(\sim \sim x \backslash \sim \sim y)$.

On the other hand, $x(x \backslash y) \leq y \leq \sim \sim y$, so $\sim \sim x \cdot \sim \sim(x \backslash y) \leq \sim \sim y$, by Lemma 2.3(4). Consequently, we have $\sim \sim(x \backslash y) \leq \sim \sim x \backslash \sim \sim y$ and $\sim(\sim \sim x \backslash \sim \sim y) \leq \sim(x \backslash y)$. Therefore, $\sim(\sim \sim x \backslash \sim \sim y)=\sim(x \backslash y)$

Additionally, we have

$$
\begin{aligned}
\sim(\sim x \vee y) & =\sim \sim \sim(\sim x \vee y) & & \text { Lemma 2.2(4)) } \\
& =\sim \sim(\sim \sim x \wedge \sim y) & & \text { Lemma } 2.2(1)) \\
& =\sim \sim(\sim y \wedge \sim \sim x) & & \\
& =\sim \sim(\sim y \cdot \sim \sim x) & & (1) \\
& =\sim(\sim \sim x \backslash \sim \sim y) & & (\text { Lemma 2.2(8)) }
\end{aligned}
$$

Consequently, $\sim(x \backslash y)=\sim(\sim x \vee y)$.

Corollary 6.9. The variety $\mathbf{G}_{\mathcal{I C} y \mathcal{F} \mathcal{L}}(\mathcal{B \mathcal { A }})$ is axiomatized relative to $\mathcal{I C} y \mathcal{F} \mathcal{L}$ by the equations:

(1) $\sim(x \wedge y) \approx \sim(x y)$

(2) $1 \leq \sim \sim(\sim \sim x \backslash x)$

(3) $1 \leq \sim \sim(x / \sim \sim x)$.

Alternatively, (1) can be replaced by either one of the equations

(4) $x \wedge \sim x \leq 0$

(5) $\sim\left(x^{2}\right) \approx \sim x$.

Proof. We will show that (1), (4) and (5) are all equivalent. First assume that (1) holds. We have $x \cdot \sim x \leq 0$, so $1 \leq(x \cdot \sim x) \backslash 0=\sim(x \cdot \sim x)=$ $\sim(x \wedge \sim x)$; hence $x \wedge \sim x \leq 0$.

Assume, now, that (4) holds. We have $x \cdot(x \backslash \sim x) \leq x$, by integrality and $x \cdot(x \backslash \sim x) \leq \sim x$. So, $x \cdot(x \backslash \sim x) \leq x \wedge \sim x \leq 0$. Therefore, $x \backslash \sim x \leq \sim x$, so $\sim\left(x^{2}\right)=x \backslash(x \backslash 0) \leq \sim x$. On the other hand, $x^{2} \leq x$, by integrality, so $\sim x \leq \sim\left(x^{2}\right)$.

Finally, if (5) holds, then we have $\sim(x \wedge y) \leq \sim(x y)$, by integrality, and $\sim(x y) \leq \sim[(x \wedge y)(x \wedge y)]=\sim(x \wedge y)^{2}=\sim(x \wedge y)$, by $(4)$.

Corollary 6.10. $\mathbf{G}_{\mathcal{C F} \mathcal{L}}(\mathcal{B A})$ is axiomatized relative to $\mathcal{C} \mathcal{F} \mathcal{L}$ by the equations:

(1) $\sim(x \wedge y) \approx \sim(x y)$

(2) $\sim(x \rightarrow y) \approx \sim(\sim x \vee y)$.

Alternatively, (2) can be replaced by 
(3) $1 \leq \sim \sim(\sim \sim x \rightarrow x)$

Corollary 6.11. The variety $\mathbf{G}_{\mathcal{I C} \mathcal{F} \mathcal{L}}(\mathcal{B A})$ is axiomatized relative to $\mathcal{I C \mathcal { F }}$ by the equations:

(1) $\sim(x \wedge y) \approx \sim(x y)$

(2) $1 \leq \sim \sim(\sim \sim x \rightarrow x)$.

Alternatively, (1) can be replaced by either one of the equations

(3) $x \wedge \sim x \leq 0$

(4) $\sim\left(x^{2}\right) \approx \sim x$.

The algebraic version of Theorem 1.1 follows from the following corollary.

Corollary 6.12. The (equational) Glivenko property holds for $\mathcal{H} \mathcal{A}$ relative to $\mathcal{B A}$.

Proof. Note that $\mathcal{H} \mathcal{A}$ satisfies $\sim \sim x \cdot x \leq x$ by integrality, so $x \leq \sim \sim x \rightarrow x$ and $\sim(\sim \sim x \rightarrow x) \leq \sim x=\sim \sim \sim x$. Consequently, $\sim \sim x \cdot \sim(\sim \sim x \rightarrow x) \leq$ $0 \leq x$, so $\sim(\sim \sim x \rightarrow x) \leq \sim \sim x \rightarrow x$, hence $\sim(\sim \sim x \rightarrow x) \wedge \sim(\sim \sim x \rightarrow$ $x) \leq 0$. Therefore, $\sim(\sim \sim x \rightarrow x) \leq 0$; thus $1 \leq \sim \sim(\sim \sim x \rightarrow x)$.

By Corollary $6.5, \mathcal{H} \mathcal{A} \subseteq \mathcal{G}$, so the equational Glivenko property holds for $\mathcal{H} \mathcal{A}$ relative to $\operatorname{In}(\mathcal{H} \mathcal{A})=\mathcal{B} \mathcal{A}$, by Theorem 5.7.

For every bounded residuated lattice $\mathbf{A}$, consider the pointed residuated lattice $\mathbf{A}^{\prime}$, that is obtained by appending to $\mathbf{A}$ a new bottom element $\perp$ and setting $0=\perp$. That $\mathbf{A}^{\prime}$ is a residuated lattice can be easily verified; alternatively it follows form [9]. Note that $\sim a=-a=0$, for all $a \in A$, and $\sim 0=-0=1$. Using Corollary 6.7, it is easy to see that $\mathbf{A}^{\prime} \in \mathbf{G}(\mathcal{B A})$. As an example we verify Corollary $6.7(2)$, for $x=0$ and $y \in A$; we have $\sim(0 \backslash y)=\sim \top=0$ and $\sim(-0 \vee y)=\sim(\top \vee y)=\sim \top=0$. Therefore, the variety $\mathbf{G}(\mathcal{B A})$ is neither integral, nor commutative, nor contractive.

6.3. The basic logic case. A basic-logic algebra or BL-algebra is an integral, commutative pointed residuated lattice that satisfies the equations

$$
0 \leq x, x(x \rightarrow y) \approx x \wedge y \text { and }(x \rightarrow y) \vee(y \rightarrow x) \approx 1 .
$$

A many-valued algebra or $M V$-algebra is an integral, commutative pointed residuated lattice that satisfies the equations $0 \leq x$ and $(x \rightarrow y) \rightarrow y \approx$ $(y \rightarrow x) \rightarrow x$. For term equivalent definitions and basic properties, see [14] and [4].

A (pointed) generalized BL-algebra or (pointed) GBL-algebra is a (pointed) residuated lattice that satisfies the equation

$$
y(y \backslash x \wedge 1) \approx x \wedge y \approx(1 \wedge x / y) y
$$

and a (pointed) generalized $M V$-algebra or (pointed) GMV-algebra is a (pointed) residuated lattice that satisfies

$$
x /(y \backslash x \wedge 1) \approx x \vee y \approx(1 \wedge x / y) \backslash x .
$$

It is not hard to see that commutative, representable (as a subdirect product of totally ordered algebras), bounded, pointed GBL-algebras in which 0 
is the least element are term equivalent to BL-algebras. Similarly, MValgebras are just commutative, bounded, pointed GMV-algebras in which 0 is the least element. In both cases, integrality follows from the fact that the algebras are bounded. For a study of GBL-algebras and GMV-algebras, representations of them and a characterization of their cancellative members, see [1] and [12]. We denote the varieties of BL-algebras, pointed GBL-

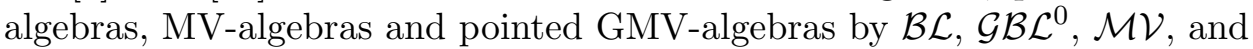
$\mathcal{G M} \mathcal{V}^{0}$, respectively. It is not difficult to see that (pointed) GMV-algebras are (pointed) GBL-algebras. Under the assumption of involutiveness the converse is true as well.

Lemma 6.13. Involutive pointed $G B L$-algebras are pointed $G M V$-algebras.

Proof. For every $x, y$ in an involutive pointed GBL-algebra, we have

$$
\begin{aligned}
x \vee y & =\sim-(x \vee y) & & (x \approx \sim-x) \\
& =\sim((-x) \wedge(-y)) & & (\text { Lemma 2.2(1)) } \\
& =\sim[(-x)((-x) \backslash(-y) \wedge 1)] & & (\mathrm{GBL}) \\
& =\sim[(-x)((\sim-x) / y \wedge 1)] & & (\text { Lemma 2.2(9)) } \\
& =\sim[-x(x / y \wedge 1)] & & (x \approx \sim-x) \\
& =(x / y \wedge 1) \backslash \sim-x & & (\text { Lemma 2.2(8)) } \\
& =(x / y \wedge 1) \backslash x & & (x \approx \sim-x) .
\end{aligned}
$$

Likewise we obtain the opposite equation.

It is observed in [5] that $\mathcal{B L}$ satisfies the equation $1 \leq \sim \sim(\sim \sim y \rightarrow y)$, and that $\mathcal{M V}$ coincides with the variety $\operatorname{In}(\mathcal{B L})$, so, by Theorem 5.7 and Corollary 6.5, Glivenko's theorem holds for $\mathcal{B L}$ relative to $\mathcal{M V}$. We will obtain a generalization by dropping the assumption of representability and by replacing the commutativity assumption by cyclicity. We first establish the following non-commutative generalization of a property observed in [5]; the proof is essentially the same.

Lemma 6.14. Every cyclic pointed GBL-algebra in which 0 is the least element satisfies the equations $1=\sim \sim(x / \sim \sim x)$ and $1=\sim \sim(\sim \sim x \backslash x)$.

Proof. First note that every pointed bounded GBL-algebra in which 0 is the least element is integral; see [1] or [12] for details. We have

$$
\sim x \cdot \sim \sim x=\sim x(\sim x \backslash 0) \leq 0 \leq x,
$$

so $\sim x \leq x / \sim \sim x$, hence $\sim(x / \sim \sim x) \leq \sim \sim x$. Consequently, we have

$$
\begin{aligned}
\sim(x / \sim \sim x) & =\sim \sim x \wedge \sim(x / \sim \sim x) & & \\
& =(\sim \sim x)((\sim \sim x) \backslash \sim(x / \sim \sim x)) & & \text { (GBL) } \\
& =(\sim \sim x) \cdot \sim((x / \sim \sim x)(\sim \sim x)) & & \text { (Lemma 2.2(8) }) \\
& =(\sim \sim x) \cdot \sim(\sim \sim x \wedge x) & & \text { (GBL) } \\
& =(\sim \sim x) \cdot \sim x \leq 0 & & \text { (Lemma 2.2(3)) }
\end{aligned}
$$

Thus, $\sim \sim(x / \sim \sim x)=1$. Similarly we prove the other equation. 
It follows from Corollary 6.9 and Lemma 6.14 that the variety $\mathcal{C} y \mathcal{G B} \mathcal{L}^{\perp}$ of cyclic pointed bounded GBL-algebras where 0 is the least element is contained in the Glivenko variety $\mathcal{G}$. Moreover, $\operatorname{In}\left(\mathcal{C} y \mathcal{G B} \mathcal{L}^{\perp}\right)$ is equal to the variety $\mathcal{C} y \mathcal{G M V}{ }^{\perp}$ of cyclic pointed bounded GMV-algebras where 0 is the least element, by Lemma 6.13 and the fact that pointed GMV-algebras are pointed GBL-algebras. Thus, in view of Theorem 5.7, we have the following corollary, which is implies Theorem ??(2).

Corollary 6.15. The (equational) Glivenko property holds for $\mathcal{C} y \mathcal{G B} \mathcal{L}^{\perp}$ relative to $\mathcal{C} y \mathcal{G} \mathcal{M V}^{\perp}$. Consequently, the (equational) Glivenko property holds for $\mathcal{B} \mathcal{L}$ relative to $\mathcal{M V}$, as well.

Following [5], a $S B L$-algebra is a BL-algebra that satisfies $x \wedge \neg x=0$. The last equation can be replaced by either of the equations $x(x \rightarrow y) \leq y$ and $\sim\left(x^{2}\right) \approx \sim x$. We denote the variety of all SBL-algebras by $\mathcal{S B} \mathcal{L}$. In [5] it is shown that the Glivenko property holds for $\mathcal{S B} \mathcal{L}$ relative to $\mathcal{B A}$, a fact that also follows from Lemma 6.14 and Corollary 6.11. We generalize this result by dropping representability and replacing commutativity with cyclicity. The following corollary is a consequence of Corollary 6.9.

Corollary 6.16. The (equational) Glivenko property holds for $\mathcal{C} y \mathcal{G B} \mathcal{L}^{\perp} \cap$ $\operatorname{Mod}\left(\sim\left(x^{2}\right) \approx \sim x\right)$ relative to $\mathcal{B} \mathcal{A}$. Consequently, the (equational) Glivenko property holds for $\mathcal{S B L}$ relative to $\mathcal{B A}$.

Note that Theorem ??(1) follows from the preceding corollary.

\section{Generalized Kolmogorov translation}

Propositional intuitionistic logic can be interpreted in propositional classical logic via the Glivenko double negation translation as well as via the Kolmogorov translation. Having studied generalizations of the former property, we now discuss the latter.

Let $\gamma$ be a unary (pointed) residuated lattice term. The $\gamma$-Kolmogorov translation $K_{\gamma}(t)$ of a (pointed) residuated lattice term $t$ is defined inductively on the complexity of $t$ as follows: $K_{\gamma}(1)=\gamma(1), K_{\gamma}(0)=\gamma(0)$, $K_{\gamma}(x)=\gamma(x)$ for every variable $x$, and $K_{\gamma}(s \star r)=\gamma\left(K_{\gamma}(s) \star K_{\gamma}(r)\right)$, where $\star \in\{\wedge, \vee, \cdot, \backslash, /\}$. Note that the standard Kolmogorov translation is obtained for $\gamma(x)=\sim \sim x$.

For every variety $\mathcal{V}$ of (pointed) residuated lattices, let $\mathcal{V}_{\gamma}$ be the subvariety of $\mathcal{V}$ axiomatized relative to $\mathcal{V}$ by the equation $\gamma(x) \approx x$. We say that the $\gamma$-Kolmogorov translation holds for $\mathcal{W}$ relative to $\mathcal{V}$, if for every set of equations $E \cup\{s \approx t\}$ in the language of (pointed) residuated lattices, $E \models_{\mathcal{V}} s \approx t$ iff $K_{\gamma}[E] \models_{\mathcal{W}} K_{\gamma}(s) \approx K_{\gamma}(t)$, where $K_{\gamma}[E]=\left\{K_{\gamma}(u) \approx\right.$ $\left.K_{\gamma}(v) \mid(u \approx v) \in E\right\}$. Also, if $\mathbf{K}$ and $\mathbf{L}$ are substructural logics, we say that the $\gamma$-Kolmogorov translation holds for $\mathbf{K}$ relative to $\mathbf{L}$, if for every set of formulas $\Phi \cup\{\psi\}$ in the language of (pointed) residuated lattices, $\Phi={ }_{\mathbf{K}} \psi$ 
iff $K_{\gamma}[\Phi] \models_{\mathbf{L}} K_{\gamma}(\psi)$, where $K_{\gamma}[\Phi]=\left\{K_{\gamma}(\phi) \mid \phi \in \Phi\right\}$. Corollary 7.6 shows the connection between these two definitions.

Note that if $\mathcal{W}$ is a subvariety of $\mathcal{F} \mathcal{L}$ and $\gamma$ is a unary (pointed) residuated lattice term, then $\gamma^{\mathbf{A}}$ is a nucleus on $\mathbf{A}$ for all $\mathbf{A} \in \mathcal{W}$ iff $\mathcal{W}$ satisfies the equations

(nuc) $\quad x \leq \gamma(x), \gamma(x) \leq \gamma(x \vee y), \gamma(\gamma(x)) \approx \gamma(x), \gamma(x) \gamma(y) \leq \gamma(x y)$

Theorem 7.1. Let $\mathcal{V}$ be a variety of (pointed) residuated lattices and $\gamma$ a term that contains only the connectives $\wedge, \backslash, /$, and also the constant 0 only if $\gamma(0) \approx 0$ holds in $\mathcal{V}$. Moreover assume that for every algebra $\mathbf{A}$ in $\mathcal{V}, \gamma^{\mathbf{A}}$ is a nucleus on $\mathbf{A}$; equivalently, assume that $\mathcal{V}$ satisfies the equations (nuc). Then, the $\gamma$-Kolmogorov translation holds for $\mathcal{V}$ relative to $\mathcal{V}_{\gamma}$.

Proof. Using induction on the length of $t$, we can show that if $t$ is a (pointed) residuated lattice term, $\mathbf{A} \in \mathcal{V}$ and $\bar{a}$ is an element of an appropriate power of $A$, then

$$
K_{\gamma}(t)^{\mathbf{A}}(\bar{a})=t^{\mathbf{A}_{\gamma}}\left(\gamma^{\mathbf{A}}(\bar{a})\right)
$$

where we have abbreviated $\mathbf{A}_{\gamma \mathbf{A}}$ to $\mathbf{A}_{\gamma}$; see Lemma 5.3 for the definition of $\mathbf{A}_{\gamma} \mathbf{A}$. To see this note that $K_{\gamma}(t)^{\mathbf{A}}(\bar{a})$ is just the application on $\gamma^{\mathbf{A}}(\bar{a})$ of the term function that corresponds to the term $t$, where every application of an operation is followed by $\gamma$; on the other hand $t^{\mathbf{A}_{\gamma}}\left(\gamma^{\mathbf{A}}(\bar{a})\right)$ is the the application on $\gamma^{\mathbf{A}}(\bar{a})$ of the term function that corresponds to the term $t$, where every operation is computed in $\mathbf{A}_{\gamma}$. The operations $\cdot, \vee$ and 1 , when computed in $\mathbf{A}_{\gamma}$ are, by definition, equal to the corresponding operations on A followed by $\gamma$. The same holds for the other operations trivially, because the result of those operations on elements of $\mathbf{A}_{\gamma}$ is already an element of $\mathbf{A}_{\gamma}$, so the application or not of $\gamma$ does not make any difference. For example, for the term $t=x \cdot y$, we have $K_{\gamma}(t)^{\mathbf{A}}(a, b)=\gamma\left(\gamma(a) \cdot{ }^{\mathbf{A}} \gamma(b)\right)$ and $t^{\mathbf{A}_{\gamma}}(\gamma(a), \gamma(b))=\gamma(a) \cdot \gamma \gamma(b)=\gamma\left(\gamma(a) \cdot \mathbf{A}_{\gamma}(b)\right)$.

Recall that $\gamma$ contains only the connectives $\wedge, \backslash, /$, and also the constant 0 only if $\gamma(0) \approx 0$ holds in $\mathcal{V}$, so $\gamma^{\mathbf{A}_{\gamma}}(a)=\gamma^{\mathbf{A}}(a)$, for every element $a$ of $\mathbf{A}_{\gamma}$. Moreover, $\gamma^{\mathbf{A}}(a)=a$, since $\gamma$ is a nucleus. Hence $\gamma^{\mathbf{A}_{\gamma}}(a)=a$, for all $a \in \mathbf{A}_{\gamma}$, and $\mathbf{A}_{\gamma} \in \mathcal{V}_{\gamma}$.

We will show that the $\gamma$-Kolmogorov translation holds for $\mathcal{V}$ relative to $\mathcal{V}_{\gamma}$. First suppose that $E \models \mathcal{V}_{\gamma} s \approx t$; we will show that $K_{\gamma}(E) \models \mathcal{V}$ $K_{\gamma}(s) \approx K_{\gamma}(t)$. Let $\mathbf{A}$ be in $\mathcal{V}$ and $\bar{a}$ be an element of an appropriate power of $A$, such that $K_{\gamma}(u)^{\mathbf{A}}(\bar{a})=K_{\gamma}(v)^{\mathbf{A}}(\bar{a})$, for all $(u \approx v) \in E$. Then, $u^{\mathbf{A}_{\gamma}}\left(\gamma^{\mathbf{A}}(\bar{a})\right)=v^{\mathbf{A}_{\gamma}}\left(\gamma^{\mathbf{A}}(\bar{a})\right)$ by $\left(^{*}\right)$, and $\mathbf{A}_{\gamma} \in \mathcal{V}_{\gamma}$. So, by assumption, $s^{\mathbf{A}_{\gamma}}\left(\gamma^{\mathbf{A}}(\bar{a})\right)=t^{\mathbf{A}_{\gamma}}\left(\gamma^{\mathbf{A}}(\bar{a})\right)$, hence $K_{\gamma}(s)^{\mathbf{A}}(\bar{a})=K_{\gamma}(t)^{\mathbf{A}}(\bar{a})$.

Conversely, if $E \not \forall_{\mathcal{V}_{\gamma}} s \approx t$, then there exists an algebra $\mathbf{B} \in \mathcal{V}_{\gamma} \subseteq \mathcal{V}$ and a sequence $\bar{b}$ of elements of $B$ such that $u^{\mathbf{B}}(\bar{b})=v^{\mathbf{B}}(\bar{b})$ for all $(u \approx v) \in E$, but $s^{\mathbf{B}}(\bar{b}) \neq t^{\mathbf{B}}(\bar{b})$. Since $\mathbf{B}$ satisfies $\gamma(x) \approx x$, we have $\mathbf{B}=\mathbf{B}_{\gamma}$. For every $(u \approx v) \in E$, we have $K_{\gamma}(u)^{\mathbf{B}}(\bar{b})=u^{\mathbf{B}_{\gamma}}\left(\gamma^{\mathbf{B}}(\bar{b})\right)=u^{\mathbf{B}}(\bar{b})=v^{\mathbf{B}}(\bar{b})=$ 
$K_{\gamma}(v)^{\mathbf{B}}(\bar{b})$ and $K_{\gamma}(s)^{\mathbf{B}}(\bar{b})=s^{\mathbf{B}}(\bar{b}) \neq t^{\mathbf{B}}(\bar{b})=K_{\gamma}(t)^{\mathbf{B}}(\bar{b})$. Consequently, $K_{\gamma}(E) \forall_{\mathcal{V}} K_{\gamma}(s) \approx K_{\gamma}(t)$.

Recall the definition of $\lambda$ from Section 5. As shown in Lemma 5.2, if we assume cyclicity, then $\lambda$ is a nucleus.

Corollary 7.2. If $\mathcal{V}$ is cyclic, then the $\lambda$-Kolmogorov translation holds for $\mathcal{V}$ relative to $\mathcal{V}_{\lambda}$.

The $\lambda$-Kolmogorov translation is simply called the Kolmogorov translation in the literature.

The following observation shows one of the differences between the Kolmogorov translation and the Glivenko property.

Theorem 7.3. Assume that $\mathcal{W}$ is a subvariety of $\mathcal{F} \mathcal{L}$, that $\gamma$ is a unary (pointed) residuated lattice term that contains only the connectives $\wedge, \backslash, /$, and also the constant 0 only if $\gamma(0) \approx 0$ holds in $\mathcal{W}$, and that $\mathcal{W}$ satisfies the equations (nuc). Then the $\gamma$-Kolmogorov translation holds for $\mathcal{W}$ relative to $\mathcal{V}$ iff $\mathcal{W}_{\gamma}=\mathcal{V}$

Proof. One direction follows from Theorem 7.1. For the forward direction, suppose that the $\gamma$-Kolmogorov translation holds for $\mathcal{W}$ relative to $\mathcal{V}$. Then, for all sets of equations $E \cup\{s \approx t\}, E \models \mathcal{V} s \approx t$ iff $K_{\gamma}(E) \models \mathcal{W} K_{\gamma}(s) \approx$ $K_{\gamma}(t)$. On the other hand, by Theorem 7.1 , we have $E \models_{\mathcal{W}_{\gamma}} s \approx t$ iff $K_{\gamma}(E) \models_{\mathcal{W}} K_{\gamma}(s) \approx K_{\gamma}(t)$. Thus, $E \models_{\mathcal{V}} s \approx t$ iff $E \models \mathcal{W}_{\gamma} s \approx t$; hence $\mathcal{V}=\mathcal{W}_{\gamma}$.

Corollary 7.4. The variety $\mathcal{I} n \mathcal{F} \mathcal{L}_{\text {ew }}$ is the only subvariety of $\mathcal{F} \mathcal{L}$ relative to which the $\lambda$-Kolmogorov translation holds for $\mathcal{F} \mathcal{L}_{\text {ew }}$.

Theorem 7.5. Assume that $\mathcal{V}$ and $\mathcal{W}$ are two subvarieties of $\mathcal{F} \mathcal{L}$ and $\gamma$ is a unary (pointed) residuated lattice term such that $\mathcal{W}$ satisfies the equations (nuc). The following are equivalent.

(1) For every set of equations $E \cup\{s \approx t\}$ in the language of (pointed) residuated lattices,

$$
E \mid=_{\mathcal{V}} s \approx t \text { iff } K_{\gamma}[E] \models_{\mathcal{W}} K_{\gamma}(s) \approx K_{\gamma}(t) .
$$

(2) For every set of formulas $\Phi \cup\{\psi\}$ in the language of (pointed) residuated lattices,

$$
\{1 \leq \phi \mid \phi \in \Phi\} \models \mathcal{V} 1 \leq \psi \text { iff }\left\{1 \leq K_{\gamma}(\phi) \mid \phi \in \Phi\right\} \mid=\mathcal{W} 1 \leq K_{\gamma}(\phi) .
$$

Proof. We first show that for every pointed residuated lattice term $s$, the variety $\mathcal{W}$ satisfies

$$
1 \leq K_{\gamma}(s) \Leftrightarrow K_{\gamma}(1)=K_{\gamma}(s \wedge 1)
$$

and

$$
1 \leq K_{\gamma}(s \backslash t) \Leftrightarrow K_{\gamma}(s) \leq K_{\gamma}(t)
$$


For $(*)$, if $1 \leq K_{\gamma}(s)$ then $K_{\gamma}(1)=\gamma(1) \leq \gamma\left(K_{\gamma}(s)\right)=K_{\gamma}(s)$, so $K_{\gamma}(1)=$ $K_{\gamma}(s) \wedge K_{\gamma}(1)$, hence $K_{\gamma}(1)=\gamma\left(K_{\gamma}(1)\right)=\gamma\left(K_{\gamma}(s) \wedge K_{\gamma}(1)\right)=K_{\gamma}(s \wedge 1)$. Conversely, if $K_{\gamma}(1)=K_{\gamma}(s \wedge 1)$, then $1 \leq \gamma(1)=K_{\gamma}(1)=K_{\gamma}(s \wedge 1)=$ $\gamma\left(K_{\gamma}(s) \wedge K_{\gamma}(1)\right) \leq \gamma\left(K_{\gamma}(s)\right)=K_{\gamma}(s)$.

For $(* *)$, we have $K_{\gamma}(s \backslash t)=\gamma\left(K_{\gamma}(s) \backslash K_{\gamma}(t)\right) \leq \gamma\left(K_{\gamma}(s)\right) \backslash \gamma\left(K_{\gamma}(t)\right)=$ $K_{\gamma}(s) \backslash K_{\gamma}(t)$, since $\mathcal{W}$ satisfies the equations (nuc), so if $1 \leq K_{\gamma}(s \backslash t)$ then $1 \leq K_{\gamma}(s) \backslash K_{\gamma}(t)$; hence $K_{\gamma}(s) \leq K_{\gamma}(t)$. Conversely, if $K_{\gamma}(s) \leq K_{\gamma}(t)$, then $1 \leq K_{\gamma}(s) \backslash K_{\gamma}(t)$; hence $1 \leq \gamma(1) \leq \gamma\left(K_{\gamma}(s) \backslash K_{\gamma}(t)\right)=K_{\gamma}(s \backslash t)$, by the definition of $K_{\gamma}$.

Assume that (1) holds. Note that $\{1 \leq \phi \mid \phi \in \Phi\} \models \mathcal{V} 1 \leq \psi$ is equivalent to $\{1 \approx 1 \wedge \phi \mid \phi \in \Phi\} \models \mathcal{V} 1 \approx 1 \wedge \psi$, and, by $(1)$, to $\left\{K_{\gamma}(1) \approx K_{\gamma}(1 \wedge \phi) \mid \phi \in\right.$ $\Phi\} \models \mathcal{W} K_{\gamma}(1) \approx K_{\gamma}(1 \wedge \psi)$. By $(*)$, this is equivalent to $\left\{1 \leq K_{\gamma}(\phi) \mid \phi \in\right.$ $\Phi\} \models \mathcal{W} 1 \leq K_{\gamma}(\phi)$.

Now, assume that (2) holds. We have $E \models \mathcal{v} s \approx t$ iff $\{1 \leq u \backslash v, 1 \leq$ $v \backslash u \mid(u \approx v) \in E\} \mid=\mathcal{V}\{1 \leq s \backslash t, 1 \leq t \backslash s\}$ iff $\left\{1 \leq K_{\gamma}(u \backslash v), 1 \leq K_{\gamma}(v \backslash u) \mid(u \approx\right.$ $v) \in E\} \models \mathcal{W}\left\{1 \leq K_{\gamma}(s \backslash t), 1 \leq K_{\gamma}(t \backslash s)\right\}$, by $(2)$, iff $\left\{K_{\gamma}(u) \leq K_{\gamma}(v), 1 \leq\right.$ $\left.K_{\gamma}(v) \leq K_{\gamma}(u) \mid(u \approx v) \in E\right\} \models \mathcal{W}\left\{K_{\gamma}(s) \leq K_{\gamma}(t), K_{\gamma}(t) \leq K_{\gamma}(s)\right\}$, by $(* *)$, iff $\left\{K_{\gamma}(u) \approx K_{\gamma}(v) \mid(u \approx v) \in E\right\} \models \mathcal{W} K_{\gamma}(s) \approx K_{\gamma}(t)$.

The following corollary is a direct consequence of Theorem 2.5 and of Theorem 7.5 .

Corollary 7.6. Let $\mathbf{K}$ and $\mathbf{L}$ be substructural logics. The $\gamma$-Kolmogorov translation holds for $\mathbf{K}$ relative to $\mathbf{L}$ iff it holds for $\mathcal{V}(\mathbf{K})$ relative to $\mathcal{V}(\mathbf{L})$.

\section{REFERENCES}

[1] P. Bahls, J. Cole, N. Galatos, P. Jipsen and C. Tsinakis, Cancellative residuated lattices, Algebra Universalis 50(1) (2003), 83-106.

[2] W. J. Blok and D. Pigozzi, Algebraizable Logics, Memoirs of the AMS 77(396), 1989.

[3] K. Blount and C. Tsinakis, The structure of residuated lattices, Internat. J. Algebra Comput. 13(4) (2003), 437-461.

[4] R. Cignoli, I. D'Otaviano and D. Mundici, Algebraic Foundations of Many-valued Reasoning, Trends in Logic - Studia Logica Library 7. Kluwer Academic Publishers, Dordrecht, 2000.

[5] R. Cignoli and A. Torrens, Hájek basic fuzzy logic and Eukasievicz infinite-valued logic, Arch. Math. Logic 42 (2003), 361-370.

[6] R. Cignoli and A. Torrens, Glivenko like theorems in natural expansions of BCK-logic, Math. Log. Quart. 50(2) (2004), 111-125.

[7] J. Czelakowski and W. Dziobiak, The parameterized local deduction theorem for quasivarieties of algebras and its application, Algebra Universalis 35(3) (1996), 373-419.

[8] J.M. Font, R. Jansana and D. Pigozzi, A survey of abstract algebraic logic, Abstract algebraic logic, Part II (Barcelona, 1997), Studia Logica 74(1-2) (2003), 13-97.

[9] N. Galatos, Minimal varieties of residuated lattices, Algebra Universalis 52(2) (2005), $215-239$.

[10] N. Galatos, Varieties of residuated lattices Ph.D. thesis, Vanderbilt University, 2003.

[11] N. Galatos and H. Ono, Algebraization, parameterized local deduction theorem and interpolation for substructural logics over FL, to appear in Studia Logica 83 (2006).

[12] N. Galatos and C. Tsinakis Generalized MV-algebras, Journal of Algebra 283(1) (2005), 254-291. 
[13] V. Glivenko, Sur quelques points de la logique de M. Brouwer, Bull. Acad. des Sci. de Belgique 15 (1929), 183-188.

[14] P. Hájek, Metamathematics of Fuzzy Logic, Kluwer, Dordrecht-Boston-London, 1998.

[15] S. Odintsov, Negative equivalence of extensions of minimal logic, Studia Logica 78 (2004), 417-442.

[16] H. Ono, Semantics for substructural logics, Substructural logics (Tübingen, 1990), 259-291, Stud. Logic Comput., 2, Oxford Univ. Press, New York, 1993.

School of Information Science, Japan Advanced Institute of Science And Technology, 1-1 Asahidai, Nomi, Ishikawa, 923-1292, Japan

E-mail address: \{galatos, ono\}@jaist.ac.jp 